\title{
The NeVAnlinna PaRAMETRIzation \\ FOR SOME INDETERMINATE STIELTJES MOMENT PROBLEMS ASSOCIATED WITH BIRTH AND DEATH PROCESSES
}

\section{Christian Berg and Galliano Valent}

\begin{abstract}
We consider two indeterminate moment problems: One corresponding to a birth and death process with quartic rates and the other corresponding to the Al-Salam-Carlitz $q$-polynomials. Using the Darboux method, we calculate their Nevanlinna matrices and several families of orthogonality measures.
\end{abstract}

\section{Introduction}

The first examples of indeterminate measures were found by Stieltjes in 1894, cf. [25] and one of his examples reads

$$
\int_{0}^{\infty} x^{n-\log x}\{1+\lambda \sin (2 \pi \log x)\} d x=\sqrt{\pi} e^{\frac{1}{4}(n+1)^{2}}, \quad n=0,1, \ldots,
$$

where $\lambda \in[-1,1]$. It gives a one-parameter family of absolutely continuous measures on $\left[0, \infty\left[\right.\right.$ which all have the same moments $s_{n}=\sqrt{\pi} \exp \left((n+1)^{2} / 4\right)$. The polynomials which are orthogonal with respect to this family of measures are a special case of the Stieltjes-Wigert polynomials, cf. [33] or [10, p. 172].

Generally speaking, polynomials corresponding to an indeterminate Hamburger moment sequence raise two important problems:

1) The description of all (positive) orthogonality measures $\nu$.

2) The characterization of those orthogonality measures $\nu$ for which the polynomials are dense in $L^{2}(\nu)$.

The first problem was solved by Nevanlinna [21], who constructed a matrix of entire functions

$$
\left(\begin{array}{ll}
A(z) & C(z) \\
B(z) & D(z)
\end{array}\right), \quad z \in \mathbb{C}
$$

with determinant identically equal to one, and showed that the Stieltjes transform of any possible orthogonality measure $\nu=\nu_{\varphi}$ is given by

$$
\int \frac{d \nu_{\varphi}(u)}{z-u}=\frac{A(z) \varphi(z)-C(z)}{B(z) \varphi(z)-D(z)}, \quad z \in \mathbb{C} \backslash \mathbb{R},
$$

where $\varphi$ is a Pick function. See Section 2 for the precise definitions.

Received July 7, 1993, revised January 3, 1994.

1991 Mathematics Subject Classification: primary 33C45; secondary 44A60.

Key words and phrases: orthogonal polynomials, birth and death processes, Al-Salam-Carlitz polynomials, indeterminate moment problems, N-extremal measures.

The first author gratefully acknowledges support from the Carlsberg Foundation. 
The second problem was solved by Marcel Riesz [22], who proved that the polynomials are dense in $L^{2}(\nu)$ precisely when $\nu$ is Nevanlinna extremal (short: N-extremal), i.e., a measure whose corresponding Pick function is constant

$$
\varphi(z)=t, \quad t \in \mathbb{R} \cup\{\infty\}
$$

By 1923, the theory of the indeterminate Hamburger moment problem was essentially accomplished, but no concrete example of this beautiful piece of work was available in the literature.

The first measure which was recognized as being $\mathrm{N}$-extremal was pointed out by Chihara for the $q$-polynomials of Al-Salam and Carlitz ([2], [10, p. 195]) without use of (1.2). For further details see [7]. Moak [20], considering $q$-analogues of Laguerre polynomials, was the first to express the functions $B, D$ in terms of known functions, viz. the $q$-Bessel functions. The $q$-Laguerre polynomials are essentially the generalized Stieltjes-Wigert polynomials, cf. [10]. Chihara and Ismail [12] have worked out the full Nevanlinna matrix (1.1) corresponding to the Al-Salam-Chihara polynomials. More recently Ismail and Masson [18] obtained the same result for the $q$-Hermite polynomials, and they have given several examples of $\mathrm{N}$-extremal as well as non- $\mathrm{N}$-extremal measures in a closed form.

In this paper we shall consider two rather different indeterminate problems. The first one has to do with the orthogonal polynomials related to a quartic birth and death process already considered in [29], [28], [7], and the second one with the Al-SalamCarlitz $q$-polynomials [2], [10], [7]. The paper is organized as follows. Section 2 gathers basic information on indeterminate Hamburger moment problems. The Nevanlinna parametrization of all the measures is described with emphasis put on the N-extremal measures. We have included a simple criterion in terms of the Pick parameter $\varphi$ for the measure $\nu_{\varphi}$ to have a continuous density (cf. 2.1.1, 2.1.2 below).

In the case of a Stieltjes moment sequence, one has to distinguish between determinacy/indeterminacy in the sense of Hamburger and in the sense of Stieltjes, where indeterminacy in the sense of Stieltjes means that there exist at least two different measures on the interval $[0, \infty$ [ with the same moments. We use the self-explanatory notation as in [7] to indicate the different cases: $\operatorname{det}(H), \operatorname{det}(S), \operatorname{indet}(H), \operatorname{indet}(S)$. For a moment sequence which is $\operatorname{indet}(S)$, we characterize the subclass of positively supported N-extremal measures $\nu_{t}$ by $t \in[\alpha, 0]$, where the constant $\alpha$ is expressed in terms of $B$ and $D$ as well as in terms of the recurrence coefficients.

Birth and death processes lead to Stieltjes moment problems, and we have gathered some useful formulas expressing fundamental quantities in terms of the birth and death rates $\left(\lambda_{n}\right),\left(\mu_{n}\right)$.

This section concludes with a detailed discussion of continuity properties of the Nevanlinna matrix and the orthogonality measures in terms of parameters on which the moment sequence depends; these results are needed in Section 4.

In Section 3-as a first example - we consider a birth and death process with quartic rates. Using generating functions determined in [30], the asymptotic analysis of the corresponding polynomials is carried through by the Darboux method and leads after long calculations to the full Nevanlinna matrix. In a preceding paper [7], $C$ and $D$ were determineci in a more elementary way. The order, type, and Phragmén-Lindelöf indicator are determined for these entire functions, e.g., the order is $\frac{1}{4}$. The present case seems to be the first, where one of the functions in the Nevanlinna matrix can be 
expressed by elementary functions, namely

$$
D(z)=\frac{4}{\pi} z^{1 / 2} \sin \left(\frac{z^{1 / 4}}{2} K_{0}\right) \sinh \left(\frac{z^{1 / 4}}{2} K_{0}\right)
$$

where $K_{0}$ is a constant.

The equation giving the support of the $\mathrm{N}$-extremal measures is derived; in two particular cases (corresponding to the endpoints of the interval $[\alpha, 0]$ ), it can be solved in closed form, and the corresponding masses are given. Several examples of non-Nextremal measures are worked out which exhibit a density and (or) discrete masses.

Using the orthogonality measures, we have expressions for the moments. This leads to a generating function for the moments already determined in [28]. We find the asymptotic behaviour of the moments by the Darboux method:

$$
s_{n}=\frac{4(4 n+1) !}{K_{0}^{4 n+2}}\left(1+O\left(\frac{1}{25^{n}}\right)\right)
$$

In Section 4, we consider the Al-Salam-Carlitz $q$-polynomials with $x$ replaced by $x+1$ in order to treat them from the point of view of a birth and death process. The birth and death rates depend on two parameters $a>0$ and $0<q<1$. The regions of $\operatorname{det}(H)$ and $\operatorname{indet}(H)$ are invariant under the transformation $a \mapsto 1 / a$. We examine how the orthogonal polynomials and the corresponding entire functions behave under this transformation. From the general study in Section 2, we know that the entire functions depend continuously on the parameters $(a, q)$ in the region of indeterminacy. This is useful in the calculations because the case $a=1$ has to be excluded in the basic calculations because of degeneracy of the formulas. The invariance properties mentioned also makes it possible to restrict the calculations to $a<1$.

Otherwise the procedure resembles that of Section 3. Using generating functions for $F_{n}$ given in [2], [7] and for $\tilde{F}_{n}$ given in [7], we apply asymptotic analysis to determine the full Nevanlinna matrix. The calculations in this case are much simpler than the quartic case because the generating functions are meromorphic, whereas they have singularities along the cut $[1, \infty[$ in the quartic case. The entire functions of the Nevanlinna matrix are all of order zero. The support of the N-extremal measures can be determined explicitly for two values of the parameter $t=0, t=t_{1}$, where

$$
t_{1}=t_{1}(a)=-1 / \xi(a), \quad \xi(a)=\frac{[q]_{\infty}}{a-1}{ }_{2} \phi_{1}\left[\begin{array}{c}
1 / a, 0 \\
q / a
\end{array} ; q\right]
$$

valid for $q<a<1 / q$. Here $[q]_{\infty}=\prod_{1}^{\infty}\left(1-q^{n}\right)$. Concerning the basic hypergeometric series ${ }_{r} \phi_{s}$, we refer to Rahman and Gasper [14], but since $q$ is fixed throughout the paper, we have omitted $q$ and write

$$
{ }_{r} \phi_{s}\left[\begin{array}{c}
a_{1}, \ldots, a_{r} \\
b_{1}, \ldots, b_{s}
\end{array} ; z\right]={ }_{r} \phi_{s}\left[\begin{array}{c}
a_{1}, \ldots, a_{r} \\
b_{1}, \ldots, b_{s}
\end{array} ; q, z\right]
$$

When the problem is $\operatorname{indet}(S)(1<a<1 / q)$ the values $t=0, t=t_{1}$ are again the endpoints of the interval $[\alpha, 0]$. When the problem is $\operatorname{det}(S)$, $\operatorname{indet}(H)(q<a \leq 1)$, we do not have this interpretation. The two values only agree in the border case $a=1$. 
The corresponding measures are

$$
\begin{gathered}
m^{(a)}=[a q]_{\infty} \sum_{n=0}^{\infty} \frac{a^{n} q^{n^{2}}}{[a q]_{n}[q]_{n}} \varepsilon_{\left(q^{-n}-1\right)} \\
\sigma^{(a)}=[q / a]_{\infty} \sum_{n=0}^{\infty} \frac{a^{-n} q^{n^{2}}}{[q / a]_{n}[q]_{n}} \varepsilon_{\left(a q^{-n}-1\right)},
\end{gathered}
$$

where $\varepsilon_{x}$ denotes the Dirac measure at $x$. By convex combinations of these measures, we obtain non- $\mathrm{N}$-extremal discrete measures. We also have calculated a one-parameter family of orthogonality measures with analytic densities.

For sake of completeness, we have calculated the unique orthogonality measure in the case $\operatorname{det}(H)$. It turns out (cf. Theorem 4.8.1) that (1.6) also gives the orthogonality measure for $0<a \leq q$, while (1.7) gives the orthogonality measure for $1 / q \leq a$. Summarizing, we have the following orthogonality measures given by (1.6) and (1.7):

$$
\begin{gathered}
m^{(a)} \text { for } 0<a<1 / q, 0<q<1 \\
\sigma^{(a)} \text { for } 0<q<a, 0<q<1 .
\end{gathered}
$$

We remark that $\sigma^{(a)}$ is concentrated on $[0, \infty[$ precisely when $a \geq 1$.

\section{The Nevanlinna parametrization}

2.1. The Hamburger case. Let us consider an indeterminate probability measure $\mu$ on the real line. The set $V$ of measures $\tau$ on $\mathbb{R}$ having the same moments as $\mu$, i.e.,

$$
s_{n}=\int x^{n} d \mu(x)=\int x^{n} d \tau(x), \quad n=0,1, \ldots,
$$

is a compact convex subset of $M_{1}(\mathbb{R})$, the set of probability measures on $\mathbb{R}$ with the weak topology, i.e., the weakest topology for which the mappings $\mu \mapsto \int f d \mu$ are continuous, when $f$ is continuous and vanishes at infinity, cf. [1, p. 31-32].

Nevanlinna [21] showed that $V$ can be parametrized by the one-point compactification $\mathcal{P} \cup\{\infty\}$ of the space $\mathcal{P}$ of Pick functions, i.e., the holomorphic functions $\varphi: \mathbb{C} \backslash \mathbb{R} \rightarrow \mathbb{C}$ given by

$$
\varphi(z)=s z+t+\int \frac{u z+1}{u-z} d \sigma(u)
$$

where $s \geq 0, t \in \mathbb{R}$ and $\sigma$ is a positive finite measure on $\mathbb{R}$. (The Pick functions form a locally compact subset of the space of holomorphic functions on $\mathbb{C} \backslash \mathbb{R}$, cf. [4].) A function of the form (2.2) clearly satisfies $\varphi(\bar{z})=\overline{\varphi(z)}$ and $\operatorname{Im} \varphi(z) \geq 0$ for $\operatorname{Im} z>0$, and holomorphic functions in $\mathbb{C} \backslash \mathbb{R}$ with these two properties have a unique representation of the form (2.2), cf. [1], [24], [26].

The parametrization is established via a homeomorphism $\varphi \mapsto \nu_{\varphi}$ of $\mathcal{P} \cup\{\infty\}$ onto $V$ given by

$$
\int \frac{d \nu_{\varphi}(u)}{z-u}=\frac{A(z) \varphi(z)-C(z)}{B(z) \varphi(z)-D(z)}, \quad z \in \mathbb{C} \backslash \mathbb{R}
$$

where $A, B, C, D$ are certain entire functions depending only on the moment sequence $\left(s_{n}\right)_{n \geq 0}$ (or equivalently on $V$ ), cf. (2.12) below. Detailed proofs can be found in [1], $[9],[24],[26]$. We follow the terminology of [1]. 
Given $\nu \in V$, we find $\varphi \in \mathcal{P} \cup\{\infty\}$ so that $\nu=\nu_{\varphi}$ by solving the equation

$$
\frac{A(z) \varphi(z)-C(z)}{B(z) \varphi(z)-D(z)}=\int \frac{d \nu(u)}{z-u} .
$$

Given $\varphi \in \mathcal{P} \cup\{\infty\}$, we find $\nu_{\varphi} \in V$ by the Stieltjes-Perron inversion procedure: We have

$$
d_{y}(x):=-\frac{1}{\pi} \operatorname{Im} \frac{A(z) \varphi(z)-C(z)}{B(z) \varphi(z)-D(z)}=P_{y} * \nu_{\varphi}(x) \quad \text { for } z=x+i y, y>0,
$$

where

$$
P_{y}(x)=\frac{1}{\pi} \frac{y}{x^{2}+y^{2}}
$$

is the Poisson kernel for the upper half-plane, and it is well-known that $\lim _{y \rightarrow 0} P_{y} * \nu_{\varphi}=$ $\nu_{\varphi}$ weakly. In other words, the measure with density $d_{y}(x)$ with respect to Lebesgue measure converges weakly to $\nu_{\varphi}$. Many standard results about harmonic functions in the upper half-plane can be applied in this situation. In particular, if $\nu_{\varphi}$ is absolutely continuous of the form $h(x) d x$, then $\lim _{y \rightarrow 0} d_{y}(x)=h(x)$ for almost all $x \in \mathbb{R}$.

In computations, the following is useful.

Proposition 2.1.1. Suppose $\varphi \in \mathcal{P}$ has boundary values

$$
\varphi\left(x^{+}\right)=\lim _{y \rightarrow 0+} \varphi(x+i y) \quad \text { for } x \in I,
$$

where $I$ is an open interval, and the convergence is uniform on compact subsets of $I$. If, furthermore, $B(x) \varphi\left(x^{+}\right)-D(x) \neq 0$ for $x \in I$, then $\nu_{\varphi}$ has a continuous density on I given by

$$
d(x)=\frac{1}{\pi} \frac{\operatorname{Im} \varphi\left(x^{+}\right)}{\left|B(x) \varphi\left(x^{+}\right)-D(x)\right|^{2}} .
$$

Proof. Using (2.13) below, we get that $\lim _{y \rightarrow 0} d_{y}(x)=d(x)$ uniformly for $x$ in compact subsets of $I$ and the assertion follows.

Remark 2.1.2. If $\varphi \in \mathcal{P}$ has the representation (2.2) with $\sigma=g(u) d u$ and $g$ is a $C^{1}$-function satisfying

$$
g^{\prime}(u)=O\left(\frac{1}{|u|^{\alpha}}\right) \text { for }|u| \rightarrow \infty, \alpha>3,
$$

then $\varphi$ has continuous boundary values

$$
\varphi\left(x^{+}\right)=s x+\tau-\pi \mathcal{H}(h)(x)+i \pi h(x), \quad x \in \mathbb{R}
$$

(the limit exists uniformly for $x$ in compact subsets of $\mathbb{R}$ ), where $\tau=t-\int u g(u) d u$, $h(u)=\left(1+u^{2}\right) g(u)$, and $\mathcal{H}$ denotes the Hilbert transform

$$
\mathcal{H}(h)(x)=\lim _{\delta \rightarrow 0} \frac{1}{\pi} \int_{|u|>\delta} \frac{h(x-u)}{u} d u,
$$

cf. (5) in [4]. Furthermore, $\operatorname{Im} \varphi\left(x^{+}\right)=\pi h(x)$, so if $g(x)>0$, then $B(x) \varphi\left(x^{+}\right)-D(x) \neq$ 0 .

To see this, one proceeds as in [4] using standard results about $\mathcal{H}$. 
The four functions in (2.3) can be defined in terms of the orthonormal polynomials $\left(P_{n}\right)_{n \geq 0}$ with respect to $\mu$, and the polynomials $\left(Q_{n}\right)_{n \geq 0}$ of the second kind given by

$$
Q_{n}(x)=\int \frac{P_{n}(x)-P_{n}(y)}{x-y} d \mu(y)
$$

We stress that $P_{n}$ is of degree $n$ with positive leading coefficient. This convention together with orthonormality determines $\left(P_{n}\right)$ uniquely.

The polynomials $P_{n}$ as well as $Q_{n}$ satisfy the three-term recurrence relation

$$
x P_{n}(x)=b_{n} P_{n+1}(x)+a_{n} P_{n}(x)+b_{n-1} P_{n-1}(x), \quad n \geq 1
$$

together with the initial conditions

$$
P_{0}(x)=1, \quad P_{1}(x)=\frac{1}{b_{0}}\left(x-a_{0}\right), \quad Q_{0}=0, \quad Q_{1}(x)=\frac{1}{b_{0}}
$$

where

$$
a_{n}=\int x P_{n}^{2}(x) d \mu(x), b_{n}=\int x P_{n}(x) P_{n+1}(x) d \mu(x), \quad n \geq 0
$$

Using (2.5) and (2.6), the following formulas are easily established

$$
\begin{aligned}
\mathcal{A}_{n}(z, w) & =(z-w) \sum_{k=0}^{n-1} Q_{k}(z) Q_{k}(w) \\
& =b_{n-1}\left(Q_{n}(z) Q_{n-1}(w)-Q_{n-1}(z) Q_{n}(w)\right) \\
\mathcal{B}_{n}(z, w) & =-1+(z-w) \sum_{k=0}^{n-1} P_{k}(z) Q_{k}(w) \\
& =b_{n-1}\left(P_{n}(z) Q_{n-1}(w)-P_{n-1}(z) Q_{n}(w)\right) \\
\mathcal{D}_{n}(z, w) & =(z-w) \sum_{k=0}^{n-1} P_{k}(z) P_{k}(w) \\
& =b_{n-1}\left(P_{n}(z) P_{n-1}(w)-P_{n-1}(z) P_{n}(w)\right)
\end{aligned}
$$

and these polynomials verify the identity

$$
\mathcal{A}_{n}(z, w) \mathcal{D}_{n}(z, w)+\mathcal{B}_{n}(z, w) \mathcal{B}_{n}(w, z)=1
$$

Because of indeterminacy the following series

$$
\sum_{k=0}^{\infty}\left|P_{k}(z)\right|^{2}, \quad \sum_{k=0}^{\infty}\left|Q_{k}(z)\right|^{2}
$$

converge uniformly on compact subsets of $\mathbb{C}$, cf. [1]. By (2.7), the polynomials $\mathcal{A}_{n}, \mathcal{B}_{n}$, 
and $\mathcal{D}_{n}$ converge uniformly on compact subsets of $\mathbb{C} \times \mathbb{C}$ to the entire functions

$$
\begin{gathered}
\mathcal{A}(z, w)=(z-w) \sum_{k=0}^{\infty} Q_{k}(z) Q_{k}(w) \\
\mathcal{B}(z, w)=-1+(z-w) \sum_{k=0}^{\infty} P_{k}(z) Q_{k}(w) \\
\mathcal{D}(z, w)=(z-w) \sum_{k=0}^{\infty} P_{k}(z) P_{k}(w)
\end{gathered}
$$

satisfying

$$
\mathcal{A}(z, w) \mathcal{D}(z, w)+\mathcal{B}(z, w) \mathcal{B}(w, z)=1
$$

Setting

$$
A(z)=\mathcal{A}(z, 0), \quad B(z)=\mathcal{B}(z, 0), \quad C(z)=-\mathcal{B}(0, z), \quad D(z)=\mathcal{D}(z, 0),
$$

we obtain the four entire functions from (2.3). They satisfy

$$
\operatorname{det}\left(\begin{array}{ll}
A(z) & C(z) \\
B(z) & D(z)
\end{array}\right)=1, \quad z \in \mathbb{C} .
$$

The matrix in (2.13) is called the Nevanlinna matrix for the indeterminate moment problem. Note that there is a change of sign in the functions in (2.10) compared with [9]. M. Riesz proved (cf. [1]) that each of the four functions $f=A, B, C, D$ is of minimal exponential type: For any $\varepsilon>0$, there exists $C_{\varepsilon}>0$ such that

$$
|f(z)| \leq C_{\varepsilon} e^{\varepsilon|z|}, \quad z \in \mathbb{C} .
$$

In other words, the order of $f$ is at most one, and if the order is one, then the type of $f$ is zero.

Recently Berg and Pedersen, cf. [5], have established that the four functions $A, B$, $C, D$ have the same order, type, and Phragmén-Lindelöf indicator function.

The solutions $\left(\nu_{t}\right)_{t \in \mathbb{R} \cup\{\infty\}}$ in $V$ corresponding to $\varphi \in \mathcal{P} \cup\{\infty\}$ being a constant $t$ (including $\infty$ ) form a remarkable class of measures called the Nevanlinna extremal solutions, (short: $N$-extremal solutions). By a theorem of M. Riesz [22], they are exactly the measures $\nu \in V$ for which the polynomials are dense in $L^{2}(\nu)$.

The entire function $B(z) t-D(z)$ has only real zeros; they are all simple and form a countable discrete set $\Lambda_{t} \subseteq \mathbb{R}$. The measure $\nu_{t}$ is concentrated in $\Lambda_{t}$, and the mass at $\lambda \in \Lambda_{t}$ is the residue

$$
\frac{A(\lambda) t-C(\lambda)}{B^{\prime}(\lambda) t-D^{\prime}(\lambda)}
$$

which is known to be equal to $\rho(\lambda)$, where

$$
\rho(z)=\left(\sum_{k=0}^{\infty}\left|P_{k}(z)\right|^{2}\right)^{-1} .
$$

The set of measures $\nu \in V$ for which the polynomials are dense in $L^{1}(\nu)$ is precisely the set of extreme points of the compact convex set $V$, cf. [1, p. 47]. We stress that $V$ is one of those infinite dimensional compact convex sets for which the extreme points are dense in $V$. For other density results about $V$, see [4]. 
A measure $\nu=\nu_{\varphi} \in V$ is called $n$-canonical, $n=0,1, \ldots$, if $\varphi \in \mathcal{P} \cup\{\infty\}$ is a rational function of degree $n$. For a rational function $\varphi=p / q$ where $p$ and $q$ are polynomials without common zeros, the degree of $\varphi$ is the maximum of the degrees of $p$ and $q$. The 0 -canonical solutions are precisely the $\mathrm{N}$-extremal solutions. By a theorem due to Buchwalter and Cassier [8], the $n$-canonical measures are precisely those $\nu \in V$ for which the closure of the polynomials in $L^{2}(\nu)$ has co-dimension $n$.

If $\varphi \in \mathcal{P}$ is a quotient of entire functions $\varphi=p / q$, in particular if $\varphi$ is rational, then we get by (2.3) that $\nu_{\varphi}$ is a discrete measure with point masses at the zeros of $B p-D q$.

If $\varphi \in \mathcal{P}$, then $\operatorname{Im} \varphi$ is a non-negative harmonic function in the upper half-plane, so by the mean-value property for harmonic functions, either $\operatorname{Im} \varphi \equiv 0$ or $\operatorname{Im} \varphi>0$ everywhere in the upper half-plane. In the first case, $\varphi$ is a real constant, and in the second case $\varphi$ maps the upper half-plane into itself and, similarly, maps the lower half-plane into itself. This shows that the class $\mathcal{P} \backslash \mathbb{R}$ of non-constant Pick functions is stable under composition. As examples of non-constant Pick functions, we mention $-1 / z, z^{\alpha}, 0<\alpha \leq 1$, and $\tan z$. In particular, if $\varphi$ is a non-constant Pick function, then so are $-(1 / \varphi), \varphi^{\alpha}, 0<\alpha \leq 1$, and $\tan \varphi$.

We next define an involution $*$ in $\mathcal{P} \cup\{\infty\}$ by

$$
\varphi^{*}=-\frac{1}{\varphi}, \quad 0^{*}=\infty, \quad \infty^{*}=0 .
$$

Via the homeomorphism $\varphi \mapsto \nu_{\varphi}$, this yields an involution $\sim$ in $V: \widetilde{\nu_{\varphi}}=\nu_{\varphi^{*}}$. The (unique) fixed point under the involution $*$ is the function

$$
\varphi_{0}(z)= \begin{cases}i, & \operatorname{Im} z>0 \\ -i, & \operatorname{Im} z<0 .\end{cases}
$$

The corresponding solution $\nu_{\varphi_{0}} \in V$ has an analytic density with respect to Lebesgue measure, cf. [4]

$$
\nu_{\varphi_{0}}=\frac{1}{\pi} \frac{1}{B^{2}(x)+D^{2}(x)} d x .
$$

More generally, corresponding to $\varphi_{t, \gamma} \in \mathcal{P}$ given by

$$
\varphi_{t, \gamma}(z)= \begin{cases}t+i \gamma, & \operatorname{Im} z>0 \\ t-i \gamma, & \operatorname{Im} z<0\end{cases}
$$

where $t \in \mathbb{R}$ and $\gamma>0$, we have

$$
\nu_{\varphi_{t, \gamma}}=\frac{\gamma}{\pi}\left\{(t B(x)-D(x))^{2}+\gamma^{2} B^{2}(x)\right\}^{-1} d x,
$$

cf. [4]. The representation (2.2) for (2.16) is given by $s=0$ and

$$
\sigma=\frac{\gamma}{\pi}\left(1+u^{2}\right)^{-1} d u
$$

We shall now relate the convex structure of $V$ to the structure of $\mathcal{P} \cup\{\infty\}$. For $\varphi, \psi \in \mathcal{P} \cup\{\infty\}$ and $s \in[0,1]$, there exists $g=g(\varphi, \psi, s) \in \mathcal{P} \cup\{\infty\}$ such that

$$
s \nu_{\varphi}+(1-s) \nu_{\psi}=\nu_{g}
$$


Inserting this into (2.3) leads to the following formula after some calculation

$$
g=\frac{\varphi \psi B-(s \varphi+(1-s) \psi) D}{((1-s) \varphi+s \psi) B-D} .
$$

In particular, using $s=\frac{1}{2}$ and constant functions $\varphi, \psi$, we get

$$
\begin{gathered}
\frac{1}{2}\left(\nu_{t}+\nu_{-t}\right)=\nu_{g}, g(z)=t^{2} \frac{B(z)}{D(z)}, \quad t \in \mathbb{R} \\
\frac{1}{2}\left(\nu_{t}+\nu_{\infty}\right)=\nu_{g}, g(z)=2 t-\frac{D(z)}{B(z)}, \quad t \in \mathbb{R} .
\end{gathered}
$$

This gives a quick proof of the fact that $B / D \in \mathcal{P}$, which may otherwise be deduced from the formula

$$
\operatorname{Im}(B(z) \overline{D(z)})=\operatorname{Im}(z) \sum_{k=0}^{\infty}\left|P_{k}(z)\right|^{2} .
$$

2.2. The Stieltjes case. In the sequel, we assume that the moment sequence (2.1) is a Stieltjes moment sequence, i.e., (2.1) has a solution $\tau$ supported by [0, $\infty[$. Since the zeros of $P_{n}$ and $Q_{n}$ are located in ]0, $\infty$ [, we see that

$$
P_{k}(x) P_{k}(0)>0, \quad P_{k}(x) Q_{k}(0)<0 \quad \text { for } x \leq 0 .
$$

If

$$
\begin{aligned}
B_{n}(x) & =\mathcal{B}_{n}(x, 0)=-1+x \sum_{k=0}^{n-1} P_{k}(x) Q_{k}(0) \\
& =b_{n-1}\left\{Q_{n-1}(0) P_{n}(x)-Q_{n}(0) P_{n-1}(x)\right\} \\
D_{n}(x) & =\mathcal{D}_{n}(x, 0)=x \sum_{k=0}^{n-1} P_{k}(x) P_{k}(0) \\
& =b_{n-1}\left\{P_{n-1}(0) P_{n}(x)-P_{n}(0) P_{n-1}(x)\right\}
\end{aligned}
$$

we see that $B_{n}(x)<B_{n+1}(x)<B(x), D_{n}(x)>D_{n+1}(x)>D(x)$ for $x<0$, and in particular for $x \leq 0$ :

$$
\begin{gathered}
B(x) \geq B_{2}(x)=-1+x Q_{1}(0) P_{1}(x)=\frac{1}{b_{0}^{2}} x\left(x-a_{0}\right)-1, \\
D(x) \leq D_{1}(x)=x
\end{gathered}
$$

It follows that the zeros $\left(\beta_{n}\right)$ and $\left(\delta_{n}\right)$ of $B$ and $D$, respectively, can be arranged in increasing order $\beta_{1}<\beta_{2}<\cdots, \delta_{1}<\delta_{2}<\cdots$ with $\beta_{1}<0, \delta_{1}=0$. From [1, p. 114], we have

$$
B_{n}^{\prime}(x) D_{n}(x)-B_{n}(x) D_{n}^{\prime}(x)=\sum_{k=0}^{n-1} P_{k}(x)^{2}
$$

hence

$$
B^{\prime}(x) D(x)-B(x) D^{\prime}(x)=\sum_{k=0}^{\infty} P_{k}(x)^{2}
$$


so that

$$
B^{\prime}\left(\beta_{n}\right) D\left(\beta_{n}\right)>0, \quad B\left(\delta_{n}\right) D^{\prime}\left(\delta_{n}\right)<0,
$$

and it follows that the zeros interlace:

$$
\beta_{1}<\delta_{1}=0<\beta_{2}<\delta_{2}<\beta_{3}<\delta_{3}<\cdots .
$$

The following result can be obtained combining [26, pp. 600-604] and [11]. We give an independent proof.

Lemma 2.2.1. The function $\varphi(x)=D(x) / B(x)$ is strictly decreasing in each of the intervals $]-\infty, \beta_{1}[,] \beta_{1}, \beta_{2}[,] \beta_{2}, \beta_{3}[, \ldots$ with

$$
\varphi\left(\beta_{j}-\right)=-\infty, \quad \varphi\left(\beta_{j}+\right)=\infty, \quad j=1,2, \ldots
$$

Furthermore

$$
\alpha:=\lim _{x \rightarrow-\infty} \frac{D(x)}{B(x)}=\lim _{n \rightarrow \infty} \frac{P_{n}(0)}{Q_{n}(0)} \leq 0 .
$$

Proof. We get

$$
\varphi^{\prime}(x)=-\frac{\sum_{k=0}^{\infty} P_{k}(x)^{2}}{B(x)^{2}}<0 \text { for } x \neq \beta_{j}, j=1,2, \ldots,
$$

and the first assertions are clear. The number

$$
\alpha:=\lim _{x \rightarrow-\infty} \frac{D(x)}{B(x)}
$$

is clearly $\leq 0$.

Using (2.23), we similarly see that $D_{n}(x) / B_{n}(x)$ is strictly decreasing in the intervals outside the zeros of $B_{n}$. By (2.22), we have

$$
\alpha_{n}:=\lim _{x \rightarrow-\infty} \frac{D_{n}(x)}{B_{n}(x)}=\frac{P_{n-1}(0)}{Q_{n-1}(0)} .
$$

The sequence $\left(\alpha_{n}\right)$ is strictly increasing since

$$
\alpha_{n+1}-\alpha_{n}=\frac{P_{n}(0) Q_{n-1}(0)-P_{n-1}(0) Q_{n}(0)}{Q_{n}(0) Q_{n-1}(0)}=\frac{-1}{b_{n-1} Q_{n}(0) Q_{n-1}(0)}>0 .
$$

Let $\chi$ be the smallest zero of $B_{2}$ and let $x<\chi$. We claim that the sequence $D_{n}(x) / B_{n}(x)$ is strictly increasing. In fact, this is equivalent to

$$
\frac{D_{n+1}(x)}{D_{n}(x)}<\frac{B_{n+1}(x)}{B_{n}(x)}
$$

which by $(2.22)$ is equivalent to

$$
\frac{D_{n}(x)}{B_{n}(x)}<\frac{P_{n}(0)}{Q_{n}(0)}
$$

but this holds by (2.26) and (2.27).

We finally get

$$
\alpha=\sup _{x<\chi} \frac{D(x)}{B(x)}=\sup _{x<\chi}\left(\sup _{n} \frac{D_{n}(x)}{B_{n}(x)}\right)=\sup _{n}\left(\sup _{x<\chi} \frac{D_{n}(x)}{B_{n}(x)}\right)=\sup _{n} \alpha_{n} .
$$


Remark 2.2.2. The number (2.25) is significant. It follows from Lemma 2.2.1 that $\operatorname{supp}\left(\nu_{t}\right) \subseteq\left[0, \infty\left[\right.\right.$ for $t \in[\alpha, 0]$, and for $t \notin[\alpha, 0]$ including $\infty$ then $\nu_{t}$ has one negative mass point. If $\alpha<0$, then the moment problem is indeterminate in the sense of Stieltjes. If $\alpha=0$, then $\nu_{0}$ is the only $\mathrm{N}$-extremal measure concentrated on $[0, \infty[$, and it turns out, cf. [11], that the moment problem is determinate in the sense of Stieltjes in this case.

For results about the density index of $\nu_{t}$ see [6].

2.3. Relation to birth and death processes. A birth and death process is defined by the sequences $\left(\lambda_{n}\right)_{n \geq 0}$ of birth rates and $\left(\mu_{n}\right)_{n \geq 0}$ of death rates, restricted by $\lambda_{n}>0, \mu_{n+1}>0$ for $n \geq 0$ and $\mu_{0} \geq 0$, cf. [17].

In order to solve Kolmogorov's equation, one has to study the polynomials $F_{n}(x)$ defined by the recurrence

$$
\left(\lambda_{n}+\mu_{n}-x\right) F_{n}(x)=\mu_{n+1} F_{n+1}(x)+\lambda_{n-1} F_{n-1}(x), \quad n \geq 0
$$

with the initial conditions

$$
F_{-1}(x)=0, \quad F_{0}(x)=1 .
$$

In addition, we shall need the polynomials $\tilde{F}_{n}(x)$ satisfying the same relations (2.28) and (2.29), but with $\left(\lambda_{n}, \mu_{n}\right)$ replaced by $\left(\lambda_{n+1}, \mu_{n+1}\right)$.

Defining

$$
\pi_{0}=1, \quad \pi_{n}=\frac{\lambda_{0} \cdots \lambda_{n-1}}{\mu_{1} \cdots \mu_{n}}, \quad n \geq 1
$$

and

$$
a_{n}=\lambda_{n}+\mu_{n}, b_{n}=\sqrt{\lambda_{n} \mu_{n+1}}, \quad n \geq 0,
$$

it is well-known that the polynomials

$$
P_{n}(x)=(-1)^{n} \frac{1}{\sqrt{\pi_{n}}} F_{n}(x), \quad Q_{n}(x)=\frac{1}{\mu_{1}}(-1)^{n-1} \frac{\tilde{F}_{n-1}(x)}{\sqrt{\pi_{n}}}
$$

satisfy (2.5) and (2.6) corresponding to a Stieltjes moment sequence (2.1).

In the following, we shall always assume $\mu_{0}=0$. Using $(2.28)$, it follows easily by induction that

$$
\begin{gathered}
F_{n}(0)=\pi_{n}, \quad n \geq 0, \\
\frac{\tilde{F}_{n-1}(0)}{\mu_{1} \pi_{n}}=\sum_{k=1}^{n} \frac{1}{\mu_{k} \pi_{k}}, \quad n \geq 1,
\end{gathered}
$$

and hence

$$
\frac{P_{n}(0)}{Q_{n}(0)}=-\left(\sum_{k=1}^{n} \frac{1}{\mu_{k} \pi_{k}}\right)^{-1}
$$

so by $(2.25)$

$$
\alpha=-\left(\sum_{k=1}^{\infty} \frac{1}{\mu_{k} \pi_{k}}\right)^{-1}
$$


The equations (2.7) can now be written

$$
\begin{gathered}
\mathcal{A}_{n}(z, w)=\lambda_{n-1} \pi_{n-1}\left(\frac{\tilde{F}_{n-2}(z)}{\mu_{1} \pi_{n-1}} \frac{\tilde{F}_{n-1}(w)}{\mu_{1} \pi_{n}}-\frac{\tilde{F}_{n-1}(z)}{\mu_{1} \pi_{n}} \frac{\tilde{F}_{n-2}(w)}{\mu_{1} \pi_{n-1}}\right) \\
\mathcal{B}_{n}(z, w)=\lambda_{n-1} \pi_{n-1}\left(\frac{F_{n}(z)}{\pi_{n}} \frac{\tilde{F}_{n-2}(w)}{\mu_{1} \pi_{n-1}}-\frac{F_{n-1}(z)}{\pi_{n-1}} \frac{\tilde{F}_{n-1}(w)}{\mu_{1} \pi_{n}}\right) \\
\mathcal{D}_{n}(z, w)=\lambda_{n-1} \pi_{n-1}\left(\frac{F_{n-1}(z)}{\pi_{n-1}} \frac{F_{n}(w)}{\pi_{n}}-\frac{F_{n}(z)}{\pi_{n}} \frac{F_{n-1}(w)}{\pi_{n-1}}\right) .
\end{gathered}
$$

To compute the entire functions $\mathcal{A}, \mathcal{B}, \mathcal{D}$ in the indeterminate case, we need the asymptotic behaviour of

$$
\lambda_{n-1} \pi_{n-1}, \quad \frac{F_{n}(z)}{\pi_{n}}, \quad \frac{\tilde{F}_{n-1}(z)}{\mu_{1} \pi_{n}}
$$

for $n \rightarrow \infty$. This will be worked out in Sections 3 and 4 for particular rates.

2.4. Parameter-dependent indeterminate problems. Suppose that the moment sequence (2.1) depends on a parameter $\gamma$ so that $\left(s_{n, \gamma}\right)_{n \geq 0}$ is a normalized indeterminate moment sequence for each $\gamma \in \Gamma$, where $\Gamma$ is a subset of a euclidean space or more generally a metric space. In the sequel, we need a continuity result in $\gamma$ for the entire functions in (2.10), and we write $P_{n, \gamma}(z), Q_{n, \gamma}(z), \mathcal{A}_{\gamma}(z, w)$, etc., for the objects corresponding to $\left(s_{n, \gamma}\right)$.

Proposition 2.4.1. Suppose that $\left(s_{n, \gamma}\right)$ is a continuous function of $\gamma$ for each $n$ and that the series

$$
\sum_{n=0}^{\infty} P_{n, \gamma}^{2}(0), \quad \sum_{n=0}^{\infty} Q_{n, \gamma}^{2}(0)
$$

converge uniformly for $\gamma$ in compact subsets of $\Gamma$. Then the functions $\mathcal{A}_{\gamma}, \mathcal{B}_{\gamma}, \mathcal{D}_{\gamma}$ from (2.10) are continuous on $\mathbb{C}^{2} \times \Gamma$.

The mapping $(\varphi, \gamma) \mapsto \nu_{\varphi, \gamma}$ from $(\mathcal{P} \cup\{\infty\}) \times \Gamma$ into $M_{1}(\mathbb{R})$ defined by

$$
\int \frac{d \nu_{\varphi, \gamma}(u)}{z-u}=\frac{A_{\gamma}(z) \varphi(z)-C_{\gamma}(z)}{B_{\gamma}(z) \varphi(z)-D_{\gamma}(z)}, \quad z \in \mathbb{C} \backslash \mathbb{R}
$$

cf. (2.3), is continuous, and, in particular, the $N$-extremal measures $\nu_{t, \gamma}$ depend continuously on $(t, \gamma) \in(\mathbb{R} \cup\{\infty\}) \times \Gamma$.

Proof. It is easy to see that $P_{n, \gamma}(z), Q_{n, \gamma}(z)$ are continuous functions of $(z, \gamma) \in \mathbb{C} \times \Gamma$ for each $n$ by the classical formulas which express $P_{n}$ and $Q_{n}$ in terms of the moments. We shall next see that the series

$$
\sum_{n=0}^{\infty}\left|P_{n, \gamma}(z)\right|^{2}, \quad \sum_{n=0}^{\infty}\left|Q_{n, \gamma}(z)\right|^{2}
$$

converge uniformly for $|z| \leq R, \gamma \in \Gamma_{0}$ for each $R>0$ and each compact subset $\Gamma_{0} \subseteq \Gamma$. From this follows the continuity of the functions in (2.38) on $\mathbb{C} \times \Gamma$ and of $\mathcal{A}_{\gamma}(z, w), \mathcal{B}_{\gamma}(z, w), \mathcal{D}_{\gamma}(z, w)$ as functions on $\mathbb{C}^{2} \times \Gamma$.

The proof that $\sum_{n=0}^{\infty}\left|P_{n, \gamma}(z)\right|^{2}$ converges uniformly in $|z| \leq R, \gamma \in \Gamma_{0}$ is a modification of the proof in [1, pp. 17-19], but, for completeness, we shall include the argument. 
We shall use the formula

$$
P_{n, \gamma}(z)=P_{n, \gamma}(0)+z \sum_{k=0}^{n-1} a_{n, k}(\gamma) P_{k, \gamma}(z), \quad n \geq 1
$$

where

$$
a_{n, k}(\gamma)=P_{k, \gamma}(0) Q_{n, \gamma}(0)-P_{n, \gamma}(0) Q_{k, \gamma}(0)
$$

are the coefficients in the orthogonal expansion of the polynomial

$$
\frac{P_{n, \gamma}(z)-P_{n, \gamma}(0)}{z} \text {. }
$$

By (2.40), we get

$$
\sum_{n=N}^{\infty} \sum_{k=0}^{n-1} a_{n, k}^{2}(\gamma) \leq 2 \sum_{n=N}^{\infty} P_{n, \gamma}^{2}(0) \sum_{k=0}^{\infty} Q_{k, \gamma}^{2}(0)+2 \sum_{n=N}^{\infty} Q_{n, \gamma}^{2}(0) \sum_{k=0}^{\infty} P_{k, \gamma}^{2}(0),
$$

and since the functions in (2.36) are continuous for $\gamma \in \Gamma$ and hence bounded on compact sets, we see that given $\varepsilon>0$ there exists $N_{0}(\varepsilon)$ such that for $\gamma \in \Gamma_{0}$

$$
\sum_{n=N_{0}(\varepsilon)}^{\infty} P_{n, \gamma}^{2}(0) \leq \frac{\varepsilon}{2}, \quad \sum_{n=N_{0}(\varepsilon)}^{\infty} \sum_{k=0}^{n-1} a_{n, k}^{2}(\gamma) \leq \frac{\varepsilon}{2 R^{2}}
$$

For $N>N_{0}(\varepsilon),|z| \leq R, \gamma \in \Gamma_{0}$, we then get from (2.39)

$$
\begin{aligned}
\sum_{n=N_{0}(\varepsilon)}^{N}\left|P_{n, \gamma}(z)\right|^{2} & \leq 2 \sum_{n=N_{0}(\varepsilon)}^{N} P_{n, \gamma}^{2}(0)+2|z|^{2} \sum_{n=N_{0}(\varepsilon)}^{N}\left|\sum_{k=0}^{n-1} a_{n, k}(\gamma) P_{k, \gamma}(z)\right|^{2} \\
& \leq \varepsilon+2 R^{2} \sum_{n=N_{0}(\varepsilon)}^{N}\left(\sum_{k=0}^{n-1} a_{n, k}^{2}(\gamma)\right)\left(\sum_{k=0}^{n-1}\left|P_{k, \gamma}(z)\right|^{2}\right) \\
& \leq \varepsilon+\varepsilon \sum_{k=0}^{N}\left|P_{k, \gamma}(z)\right|^{2}
\end{aligned}
$$

and hence

$$
(1-\varepsilon) \sum_{n=N_{0}(\varepsilon)}^{N}\left|P_{n, \gamma}(z)\right|^{2} \leq \varepsilon+\varepsilon \sum_{k=0}^{N_{0}(\varepsilon)-1}\left|P_{k, \gamma}(z)\right|^{2} .
$$

Choosing $\varepsilon=\frac{1}{2}$, we get for $N>N_{1}:=N_{0}\left(\frac{1}{2}\right),|z| \leq R, \gamma \in \Gamma_{0}$

$$
\sum_{n=N_{1}}^{N}\left|P_{n, \gamma}(z)\right|^{2} \leq 1+\sum_{n=0}^{N_{1}-1}\left|P_{n, \gamma}(z)\right|^{2}
$$

By continuity

$$
K:=\sup _{|z| \leq R, \gamma \in \Gamma_{0}} \sum_{n=0}^{N_{1}-1}\left|P_{n, \gamma}(z)\right|^{2}<\infty
$$


but this shows that the series $\sum\left|P_{n, \gamma}(z)\right|^{2}$ converges pointwise and satisfies

$$
\sum_{n=0}^{\infty}\left|P_{n, \gamma}(z)\right|^{2}=\sum_{n=0}^{N_{1}-1}\left|P_{n, \gamma}(z)\right|^{2}+\sum_{n=N_{1}}^{\infty}\left|P_{n, \gamma}(z)\right|^{2} \leq 1+2 K
$$

for $|z| \leq R, \gamma \in \Gamma_{0}$. By (2.41), we finally get for $N>N_{0}(\varepsilon)$

$$
\sum_{n=N_{0}(\varepsilon)}^{N}\left|P_{n, \gamma}(z)\right|^{2} \leq \frac{\varepsilon}{1-\varepsilon}(2+2 K) \text { for }|z| \leq R, \gamma \in \Gamma_{0},
$$

which shows the uniform convergence for $|z| \leq R, \gamma \in \Gamma_{0}$.

From (2.39), we find

$$
Q_{n, \gamma}(z)=Q_{n, \gamma}(0)+z \sum_{k=0}^{n-1} a_{n, k}(\gamma) Q_{k, \gamma}(z)
$$

from which we similarly see that $\sum\left|Q_{n, \gamma}(z)\right|^{2}$ converges uniformly for $|z| \leq R, \gamma \in \Gamma_{0}$.

Concerning the last statement, let $\left(\varphi_{n}, \gamma_{n}\right) \rightarrow(\varphi, \gamma)$ in $(\mathcal{P} \cup\{\infty\}) \times \Gamma$ and let $\nu$ be a weak accumulation point for the sequence $\left(\nu_{\varphi_{n}, \gamma_{n}}\right)$. By $(2.37)$, we then have

$$
\int \frac{d \nu(u)}{z-u}=\frac{A_{\gamma}(z) \varphi(z)-C_{\gamma}(z)}{B_{\gamma}(z) \varphi(z)-D_{\gamma}(z)}, \quad z \in \mathbb{C} \backslash \mathbb{R},
$$

showing that $\nu=\nu_{\varphi, \gamma}$ by unicity of the Stieltjes transformation. Since any weak accumulation point for the sequence $\left(\nu_{\varphi_{n}, \gamma_{n}}\right)$ is equal to $\nu_{\varphi, \gamma}$, the latter is the weak limit of the sequence.

Remark 2.4.2. With the above terminology, the recurrence coefficients from (2.5) can be written $a_{n, \gamma}, b_{n, \gamma}$. To require that $s_{n, \gamma}$ is continuous in $\gamma$ for each $\mathrm{n}$ is equivalent to the requirement that $a_{n, \gamma}$ and $b_{n, \gamma}$ are continuous in $\gamma$ for each $n$. Furthermore, if the moment problem corresponds to a birth and death process with rates $\lambda_{n, \gamma}, \mu_{n, \gamma}$, then continuity in $\gamma$ of these rates implies continuity of $s_{n, \gamma}$ for each $n$ by (2.31).

\section{A quartic birth and death process}

We shall consider the following quartic rates

$$
\lambda_{n}=(4 n+1)(4 n+2)^{2}(4 n+3), \mu_{n}=(4 n-1)(4 n)^{2}(4 n+1), \quad n \geq 0,
$$

already considered in [19], [28], [29]. Note that $\mu_{0}=0$ and

$$
\pi_{n}=\frac{1}{4 n+1}\left(\frac{(1 / 2)_{n}}{n !}\right)^{2} \sim \frac{1}{4 \pi} \frac{1}{n^{2}}, \quad \lambda_{n-1} \pi_{n-1}=\mu_{n} \pi_{n} \sim \frac{64}{\pi} n^{2},
$$

and it follows from known criteria that the corresponding moment problem is indet $(S)$, cf. [7]. 
3.1. The generating functions. The polynomials $F_{n}(z), \tilde{F}_{n}(z)$ corresponding to the rates (3.1) have the generating functions

$$
\begin{gathered}
\sum_{n=0}^{\infty} \frac{n !}{(1 / 2)_{n}} F_{n}(z) w^{n}=\frac{\delta_{1}\left(z^{1 / 4} \theta\left(w^{1 / 4}\right)\right)}{(z w)^{1 / 4}} \\
\sum_{n=0}^{\infty} \frac{(n+1) !}{(1 / 2)_{n+1}} \frac{\tilde{F}_{n}(z)}{\mu_{1}} w^{4 n+5}=\frac{1}{6} \int_{0}^{\theta(w)} t(u)^{3} \frac{\delta_{1}\left(z^{1 / 4}(\theta(w)-u)\right)}{z^{1 / 4}} d u \\
+2 \int_{0}^{\theta(w)} t(u)^{5} \frac{\delta_{3}\left(z^{1 / 4}(\theta(w)-u)\right)}{z^{3 / 4}} d u
\end{gathered}
$$

valid for $z \in \mathbb{C}, w \in \mathbb{C},|w|<1$. These formulas are special cases of the generating function (54) in [30]. The first corresponds to $c=0, \mu=0$ and the second to $c=1$, $\mu=0$.

Let us briefly recall the terminology employed above. The formula

$$
\theta(w)=\int_{0}^{w} \frac{d u}{\sqrt{1-u^{4}}}
$$

defines a conformal mapping of $\mathbb{C} \backslash \bigcup_{k=0}^{3} i^{k}\left[1, \infty\left[\right.\right.$ onto the square with corners $\pm \frac{K_{0}}{\sqrt{2}}$ $\pm i \frac{K_{0}}{\sqrt{2}}$, where

$$
\frac{K_{0}}{\sqrt{2}}=\int_{0}^{1} \frac{d u}{\sqrt{1-u^{4}}}=\frac{\Gamma\left(\frac{1}{4}\right)^{2}}{4 \sqrt{2 \pi}}
$$

is the complete elliptic integral in the lemniscatic case corresponding to the modulus $k=1 / \sqrt{2}$. The inverse function is

$$
w=t(\theta)=\frac{\operatorname{sd}(\sqrt{2} \theta)}{\sqrt{2}}=\frac{1}{\sqrt{2}} \frac{\operatorname{sn}(\sqrt{2} \theta)}{\operatorname{dn}(\sqrt{2} \theta)},
$$

where we follow the terminology of [32] omitting $k$ in the notation.

The trigonometric functions of order 4 , cf. [13], are entire and given by

$$
\delta_{l}(z)=\sum_{n=0}^{\infty}(-1)^{n} \frac{z^{4 n+l}}{(4 n+l) !}, \quad l=0,1,2,3 .
$$

(Notice that $\delta_{l}$ here differs from $\delta_{l}$ in [30] by a factor $\exp \left(i l \frac{\pi}{4}\right)$.) Since these functions play a prominent role in our analysis, let us give their most useful properties. Their derivatives are

$$
\delta_{0}^{\prime}=-\delta_{3}, \quad \delta_{1}^{\prime}=\delta_{0}, \quad \delta_{2}^{\prime}=\delta_{1}, \quad \delta_{3}^{\prime}=\delta_{2}
$$

from which we deduce $\delta_{l}^{(4)}+\delta_{l}=0, l=0,1,2,3$, which explains their name. Putting $j=\exp \left(i \frac{\pi}{4}\right)$, one can write the functions as linear combinations of the exponentials 
$\exp (\tau z), \tau \in\{ \pm j, \pm \bar{j}\}, \mathrm{cf}$. [30], and the simplest cases are

$$
\begin{aligned}
\delta_{0}(z) & =\frac{1}{4}\left(e^{j z}+e^{-j z}+e^{\bar{j} z}+e^{-\bar{j} z}\right)=\cos \left(\frac{z}{\sqrt{2}}\right) \cosh \left(\frac{z}{\sqrt{2}}\right) \\
& =\frac{1}{2}(\cosh (j z)+\cosh (\bar{j} z)), \\
\delta_{2}(z) & =\frac{1}{4 i}\left(e^{j z}+e^{-j z}-e^{\bar{j} z}-e^{-\bar{j} z}\right)=\sin \left(\frac{z}{\sqrt{2}}\right) \sinh \left(\frac{z}{\sqrt{2}}\right) \\
& =\frac{1}{2 i}(\cosh (j z)-\cosh (\bar{j} z)) .
\end{aligned}
$$

These functions satisfy addition theorems; for instance,

$$
\delta_{0}(z+w)=\delta_{0}(z) \delta_{0}(w)-\delta_{2}(z) \delta_{2}(w)-\delta_{1}(z) \delta_{3}(w)-\delta_{3}(z) \delta_{1}(w)
$$

and similar results for $l=1,2,3$.

Due to the special form of $\delta_{l}$ it is seen that we do not have to worry about which branch of the fourth root we are using in (3.3) and (3.4). We shall now transform these formulas.

Proposition 3.1.1. The following generating functions hold for $z \in \mathbb{C},|w|<1$

$$
\begin{gathered}
\sum_{n=0}^{\infty}(4 n+1) \frac{n !}{(1 / 2)_{n}} F_{n}(z) w^{n}=\frac{\delta_{0}\left(z^{1 / 4} \theta\left(w^{1 / 4}\right)\right)}{\sqrt{1-w}} \\
\sum_{n=0}^{\infty}(4 n+5) \frac{(n+1) !}{(1 / 2)_{n+1}} \frac{\tilde{F}_{n}(z)}{\mu_{1}} w^{n+1} \\
=\frac{1}{\sqrt{1-w}} \int_{0}^{\theta\left(w^{1 / 4}\right)} \frac{\delta_{2}\left(z^{1 / 4}\left(\theta\left(w^{1 / 4}\right)-u\right)\right)}{z^{1 / 2}} \frac{\operatorname{sd}(\sqrt{2} u)}{\sqrt{2}} d u .
\end{gathered}
$$

The right hand side of these equations are holomorphic in $\mathbb{C} \backslash[1, \infty[$.

Proof. Applying the operator $w d / d w+1 / 4$ to (3.3), we get (3.13).

Integrating by parts twice in (3.4) and using the identity

$$
2 t(\theta)^{5}=t(\theta)-\frac{d^{2}}{d \theta^{2}}\left(\frac{t(\theta)^{3}}{3 !}\right)
$$

where $t(\theta)$ is given by (3.7), we get

$$
\sum_{n=0}^{\infty} \frac{(n+1) !}{(1 / 2)_{n+1}} \frac{\tilde{F}_{n}(z)}{\mu_{1}} w^{4 n+5}=\int_{0}^{\theta(w)} \frac{\delta_{3}\left(z^{1 / 4}(\theta(w)-u)\right)}{z^{3 / 4}} \frac{\operatorname{sd}(\sqrt{2} u)}{\sqrt{2}} d u
$$

Differentiating with respect to $w$ and replacing $w^{4}$ by $w$ leads to (3.14). 
3.2. Asymptotic analysis. The essential tool in the asymptotic analysis of $F_{n}(z)$, $\tilde{F}_{n}(z)$ for $n \rightarrow \infty$ is the Darboux theorem [27, p. 207], which states that the large $n$ behaviour is controlled by the nearest singularity of the corresponding generating function, here $w=1$, which is the only singularity on $|w|=1$.

Let us start with (3.13). The change of variable $v=u^{4} / w$ in (3.5) gives

$$
\theta\left(w^{1 / 4}\right)=\frac{w^{1 / 4}}{4} \int_{0}^{1} \frac{d v}{v^{3 / 4} \sqrt{1-v w}}=w^{1 / 4}{ }_{2} F_{1}\left[\begin{array}{c}
1 / 2,1 / 4 \\
5 / 4
\end{array} ; w\right]
$$

by Euler's integral representation of the hypergeometric function. We transform the hypergeometric function to the variable $1-w$ using [15, formula 9.131(2)] and get

$$
\theta\left(w^{1 / 4}\right)=\frac{K_{0}}{\sqrt{2}}-\frac{1}{2} w^{1 / 4}(1-w)_{2}^{1 / 2} F_{1}\left[\begin{array}{c}
1,3 / 4 \\
3 / 2
\end{array} ; 1-w\right],
$$

valid for $|w|<1,|1-w|<1$, and the right-hand side gives the analytic extension of $\theta\left(w^{1 / 4}\right)$ in $\left.\left.\mathbb{C} \backslash\right]-\infty, 1\right]$. Using $w^{1 / 4}=(1-(1-w))^{1 / 4}$, we therefore find for $w$ close to 1

$$
\theta\left(w^{1 / 4}\right)=\frac{K_{0}}{\sqrt{2}}+(1-w)^{1 / 2}\left(-\frac{1}{2}-\frac{1}{8}(1-w)+\cdots\right) .
$$

Putting $\tilde{z}=z^{1 / 4} K_{0} / \sqrt{2}$ and $u=z^{1 / 4}\left(-\frac{1}{2}-\frac{1}{8}(1-w)+\cdots\right)$, the Taylor expansion of $\delta_{0}$ in a neighbourhood of $\tilde{z}$ gives

$$
\begin{aligned}
\frac{\delta_{0}\left(z^{1 / 4} \theta\left(w^{1 / 4}\right)\right)}{\sqrt{1-w}}= & \sum_{k=0}^{\infty} \frac{\delta_{0}^{(k)}(\tilde{z})}{k !} u^{k}(1-w)^{(k-1) / 2} \\
= & (1-w)^{-1 / 2} \delta_{0}(\tilde{z})+(1-w)^{1 / 2} \frac{\delta_{0}^{(2)}(\tilde{z})}{2 !} u^{2} \\
& +(1-w)^{3 / 2} \frac{\delta_{0}^{(4)}(\tilde{z})}{4 !} u^{4}+\cdots+\varphi(w),
\end{aligned}
$$

where $\varphi$ is the sum corresponding to odd $k$ which is holomorphic in a neighbourhood of 1 . Using the expression for $u$ and (3.9), we find the above is equal to

$$
\begin{aligned}
& (1-w)^{-1 / 2} \delta_{0}(\tilde{z})-\frac{1}{8}(1-w)^{1 / 2} z^{1 / 2} \delta_{2}(\tilde{z}) \\
& \quad-\frac{1}{16}(1-w)^{3 / 2}\left[z^{1 / 2} \delta_{2}(\tilde{z})+\frac{z}{24} \delta_{0}(\tilde{z})\right]+\cdots+\varphi(w) .
\end{aligned}
$$

Inserting the expansion

$$
(1-w)^{a}=\sum_{n=0}^{\infty} \frac{(-a)_{n}}{n !} w^{n}
$$

for $a=-\frac{1}{2}, \frac{1}{2}, \frac{3}{2}$ and collecting the coefficients to $w^{n}$, we finally get the following asymptotic expansion

$$
\begin{aligned}
\frac{F_{n}(z)}{\pi_{n}}=\delta_{0} & (\tilde{z})+\frac{1 / 8}{2 n-1} z^{1 / 2} \delta_{2}(\tilde{z}) \\
& \quad-\frac{3 / 16}{(2 n-1)(2 n-3)}\left[z^{1 / 2} \delta_{2}(\tilde{z})+\frac{z}{24} \delta_{0}(\tilde{z})\right]+O\left(\frac{1}{n^{3}}\right) .
\end{aligned}
$$


In the asymptotic analysis of (3.14), we need the following entire functions

$$
\Delta_{l}(z)=\frac{K_{0}}{\sqrt{2}} \int_{0}^{1} \delta_{l}(u z) \operatorname{cn}\left(K_{0} u\right) d u, \quad l=0,1,2,3 .
$$

We make the substitution $t=\sqrt{2}\left(\theta\left(w^{1 / 4}\right)-u\right)$, put $\sigma=\sigma(w)=K_{0}-\sqrt{2} \theta\left(w^{1 / 4}\right)$ for $|w|<1$, and transform the right-hand side of (3.14) to

$$
\frac{z^{-\frac{1}{2}}}{\sqrt{2(1-w)}} \int_{0}^{K_{0}-\sigma(w)} \delta_{2}\left(z^{1 / 4} \frac{t}{\sqrt{2}}\right) \operatorname{cn}(\sigma(w)+t) d t
$$

We next write this as $I_{0}-I_{1}$, where

$$
\begin{gathered}
I_{0}=\frac{z^{-\frac{1}{2}}}{\sqrt{2(1-w)}} \int_{0}^{K_{0}} \delta_{2}\left(z^{1 / 4} \frac{t}{\sqrt{2}}\right) \operatorname{cn}(\sigma(w)+t) d t \\
I_{1}=\frac{z^{-\frac{1}{2}}}{\sqrt{2(1-w)}} \int_{K_{0}-\sigma(w)}^{K_{0}} \delta_{2}\left(z^{1 / 4} \frac{t}{\sqrt{2}}\right) \operatorname{cn}(\sigma(w)+t) d t .
\end{gathered}
$$

From (3.16), we get

$$
\sigma=\sqrt{2(1-w)}\left(\frac{1}{2}+\frac{1}{8}(1-w)+\cdots\right)
$$

a) Asymptotic behaviour of $I_{0}$. We use the Taylor expansion of $\mathrm{cn}$ at the point $t$ and get

$$
I_{0}=\sum_{k=0}^{\infty} \frac{z^{-1 / 2}}{\sqrt{2(1-w)}} \frac{\sigma(w)^{2 k}}{(2 k) !} \int_{0}^{K_{0}} \delta_{2}\left(z^{1 / 4} \frac{t}{\sqrt{2}}\right) \operatorname{cn}^{(2 k)}(t) d t+\varphi_{1}(w)
$$

where the odd powers sum to a function $\varphi_{1}$ which is holomorphic in a neighbourhood of 1 .

The integrals

$$
c_{k}=\int_{0}^{K_{0}} \delta_{2}\left(z^{1 / 4} \frac{t}{\sqrt{2}}\right) \mathrm{cn}^{(2 k)}(t) d t
$$

can be evaluated by means of the functions $\Delta_{l}$ in (3.18) using integration by parts. Putting $\tilde{z}=z^{1 / 4} K_{0} / \sqrt{2}$ as before, we find for $k=0,1,2$, which is enough to get an expansion which is $O\left(1 / n^{3}\right)$ :

$$
\begin{gathered}
c_{0}=\sqrt{2} \Delta_{2}(\tilde{z}) \\
c_{1}=-\frac{1}{\sqrt{2}} \delta_{2}(\tilde{z})+\frac{z^{1 / 2}}{\sqrt{2}} \Delta_{0}(\tilde{z}) \\
c_{2}=-\frac{z^{1 / 2}}{2 \sqrt{2}} \delta_{0}(\tilde{z})-\frac{z}{2 \sqrt{2}} \Delta_{2}(\tilde{z}) .
\end{gathered}
$$


b) Asymptotic behaviour of $I_{1}$. Defining

$$
H(t)=\delta_{2}\left(z^{1 / 4} \frac{t}{\sqrt{2}}\right) \operatorname{cn}(\sigma+t)=\sum_{k=0}^{\infty} \frac{\mathrm{cn}^{(k)}(t)}{k !} \sigma^{k} \delta_{2}\left(z^{1 / 4} \frac{t}{\sqrt{2}}\right)
$$

and using the Taylor series for $H$ at $K_{0}$, we get

$$
I_{1}=\frac{z^{-1 / 2}}{\sqrt{2(1-w)}} \sum_{j=0}^{\infty}(-1)^{j} \frac{H^{(j)}\left(K_{0}\right)}{(j+1) !} \sigma^{j+1},
$$

where

$$
H^{(j)}\left(K_{0}\right)=\sum_{k=0}^{\infty} \frac{\sigma^{k}}{k !} \sum_{p=0}^{j}\left(\begin{array}{l}
j \\
p
\end{array}\right) \mathrm{cn}^{(k+p)}\left(K_{0}\right) \delta_{2}^{(j-p)}(\tilde{z})\left(\frac{z^{1 / 4}}{\sqrt{2}}\right)^{j-p} .
$$

Now $\mathrm{cn}^{(j)}\left(K_{0}\right)=0$ for $j \not \equiv 4 n+1, \mathrm{cn}^{\prime}\left(K_{0}\right)=-1 / \sqrt{2}$, and we need only consider even powers $\leq 4$ of $\sigma$ in order to find an expansion which is $O\left(1 / n^{3}\right)$. After some calculation, we find

$$
I_{1}=\frac{z^{-1 / 2}}{\sqrt{2(1-w)}}\left(-\frac{1}{2 \sqrt{2}} \delta_{2}(\tilde{z}) \sigma^{2}-\frac{z^{1 / 2}}{48 \sqrt{2}} \delta_{0}(\tilde{z}) \sigma^{4}+\cdots\right)+\varphi_{2}(w)
$$

and hence

$$
\begin{aligned}
I_{0}-I_{1}= & z^{-1 / 2}(1-w)^{-1 / 2} \Delta_{2}(\tilde{z})+\frac{1}{8}(1-w)^{1 / 2} \Delta_{0}(\tilde{z}) \\
& +\frac{1}{16}(1-w)^{3 / 2}\left(\Delta_{0}(\tilde{z})-\frac{z^{1 / 2}}{24} \Delta_{2}(\tilde{z})\right)+\cdots+\varphi_{3}(w),
\end{aligned}
$$

where $\varphi_{2}, \varphi_{3}$ are holomorphic functions in a neighbourhood of 1 .

Using the expansion of $(1-w)^{a}$ as before, we finally get

$$
\begin{aligned}
\frac{\tilde{F}_{n-1}(z)}{\mu_{1} \pi_{n}}= & z^{-1 / 2} \Delta_{2}(\tilde{z})-\frac{1 / 8}{2 n-1} \Delta_{0}(\tilde{z}) \\
& +\frac{3 / 16}{(2 n-1)(2 n-3)}\left(\Delta_{0}(\tilde{z})-\frac{z^{1 / 2}}{24} \Delta_{2}(\tilde{z})\right)+O\left(\frac{1}{n^{3}}\right) .
\end{aligned}
$$

3.3. The Nevanlinna matrix. Plugging (3.2), (3.17), and (3.21) into (2.35) and letting $n \rightarrow \infty$ leads after some computation to the following result.

Proposition 3.3.1. The entire functions (2.10) are given for $(z, w) \in \mathbb{C}^{2}$ by

$$
\begin{gathered}
\mathcal{A}(z, w)=\frac{4}{\pi}\left(\frac{1}{\sqrt{z}} \Delta_{2}(\tilde{z}) \Delta_{0}(\tilde{w})-\Delta_{0}(\tilde{z}) \frac{1}{\sqrt{w}} \Delta_{2}(\tilde{w})\right) \\
\mathcal{B}(z, w)=-\frac{4}{\pi}\left(\delta_{0}(\tilde{z}) \Delta_{0}(\tilde{w})+\sqrt{z} \delta_{2}(\tilde{z}) \frac{1}{\sqrt{w}} \Delta_{2}(\tilde{w})\right) \\
\mathcal{D}(z, w)=\frac{4}{\pi}\left(\sqrt{z} \delta_{2}(\tilde{z}) \delta_{0}(\tilde{w})-\delta_{0}(\tilde{z}) \sqrt{w} \delta_{2}(\tilde{w})\right)
\end{gathered}
$$

where, for simplicity,

$$
\tilde{z}=\frac{z^{1 / 4}}{\sqrt{2}} K_{0}, \quad \tilde{w}=\frac{w^{1 / 4}}{\sqrt{2}} K_{0} .
$$

(Note that $\Delta_{2}(\tilde{z}) / \sqrt{z}$ and $\sqrt{z} \delta_{2}(\tilde{z})$ are entire functions in $z$ ). 
From (3.18), we find

$$
\begin{gathered}
\Delta_{0}(0)=\frac{1}{\sqrt{2}} \int_{0}^{K_{0}} \operatorname{cn} u d u=\frac{\pi}{4}, \\
\xi:=\lim _{z \rightarrow 0} \frac{1}{\sqrt{z}} \Delta_{2}(\tilde{z})=\frac{1}{4 \sqrt{2}} \int_{0}^{K_{0}} u^{2} \operatorname{cn} u d u \approx 0.12 .
\end{gathered}
$$

Proposition 3.3.2. The Nevanlinna matrix consists of the following entire functions

$$
\begin{aligned}
& A(z)=\frac{1}{\sqrt{z}} \Delta_{2}(\tilde{z})-\frac{4}{\pi} \xi \Delta_{0}(\tilde{z}), \quad C(z)=\frac{4}{\pi} \Delta_{0}(\tilde{z}), \\
& B(z)=-\delta_{0}(\tilde{z})-\frac{4}{\pi} \xi \sqrt{z} \delta_{2}(\tilde{z}), \quad D(z)=\frac{4}{\pi} \sqrt{z} \delta_{2}(\tilde{z}),
\end{aligned}
$$

where $\tilde{z}=z^{1 / 4} K_{0} / \sqrt{2}$.

They are all of order $1 / 4$ and type $K_{0} / \sqrt{2}$, and they have the common PhragménLindelöf indicator

$$
h(\theta)=\frac{K_{0}}{2}\left(\left|\cos \frac{\theta}{4}\right|+\left|\sin \frac{\theta}{4}\right|\right), \quad \theta \in \mathbb{R} .
$$

For $D$ we have the following expressions

$$
D(z)=\frac{4}{\pi} \sqrt{z} \sin \left(\frac{z^{1 / 4}}{2} K_{0}\right) \sinh \left(\frac{z^{1 / 4}}{2} K_{0}\right)=\frac{K_{0}^{2}}{\pi} z \prod_{n=1}^{\infty}\left(1-\frac{z}{\left(2 n \pi / K_{0}\right)^{4}}\right) .
$$

Proof. It has been proved in [5] that all four functions in a Nevanlinna matrix have the same order, type, and indicator. Therefore, it is sufficient to study the simplest of them, namely $D$. Using the formula (3.11) for $\delta_{2}$ and the canonical products for sin and $\sinh$, we find (3.25). It follows that the order $\rho_{D}$ of $D$ is equal to the exponent of convergence of the zeros $\left(2 n \pi / K_{0}\right)^{4}$, i.e., the smallest number $\tau>0$ such that

$$
\sum_{n=1}^{\infty} \frac{1}{\left(2 n \pi / K_{0}\right)^{4 \tau}}<\infty \text {. }
$$

Hence, we conclude that $\rho_{D}=\frac{1}{4}$. In order to get the type, it is easier to compute first the indicator function $h_{D}$ defined by

$$
h_{D}(\theta)=\limsup _{r \rightarrow \infty} \frac{\log \left|D\left(r e^{i \theta}\right)\right|}{r^{\rho_{D}}} .
$$

Using (3.25), we find for $z=r e^{i \theta}, \theta \in[0,2 \pi[$

$$
\left|D\left(r e^{i \theta}\right)\right|=\frac{4}{\pi} \sqrt{r}\left(\left(\sinh ^{2} u+\sin ^{2} v\right)\left(\sinh ^{2} v+\sin ^{2} u\right)\right)^{1 / 2},
$$

where $u=\frac{1}{2} K_{0} r^{1 / 4} \sin \frac{\theta}{4}, v=\frac{1}{2} K_{0} r^{1 / 4} \cos \frac{\theta}{4}$, and we get

$$
h_{D}(\theta)=\frac{1}{2} K_{0}\left(\sin \frac{\theta}{4}+\cos \frac{\theta}{4}\right), \quad \theta \in[0,2 \pi[,
$$

which shows that $h_{D}$ as periodic function with period $2 \pi$ is given by (3.24). Therefore, the type $\sigma_{D}$ is

$$
\sigma_{D}=\sup _{\theta} h_{D}(\theta)=\frac{K_{0}}{\sqrt{2}}
$$


Corollary 3.3.3. The numbers $\xi$, $\alpha$ given by (3.23) and (2.25) are connected by

$$
\alpha \xi=-1
$$

which leads to the formula

$$
{ }_{4} F_{3}\left[\begin{array}{c}
1,1,1,3 / 4 \\
3 / 2,3 / 2,7 / 4
\end{array} ; 1\right]=\frac{3}{\sqrt{2}} \int_{0}^{K_{0}} u^{2} \operatorname{cn} u d u \quad(=12 \xi) .
$$

Proof. We have

$$
\frac{B(z)}{D(z)}=-\xi-\frac{\pi}{4 \sqrt{z}} \frac{\delta_{0}(\tilde{z})}{\delta_{2}(\tilde{z})}
$$

and for $z=-x, x>0$, this leads by (3.10) and (3.11) to

$$
\frac{B(-x)}{D(-x)}=-\xi+\frac{\pi}{4 \sqrt{x}} i \frac{\delta_{0}(j \tilde{x})}{\delta_{2}(j \tilde{x})}=-\xi+\frac{\pi}{4 \sqrt{x}} \frac{\cosh (\tilde{x})+\cos (\tilde{x})}{\cosh (\tilde{x})-\cos (\tilde{x})}
$$

with $\tilde{x}=\frac{x^{1 / 4}}{\sqrt{2}} K_{0}$, and we get

$$
\frac{1}{\alpha}=\lim _{x \rightarrow \infty} \frac{B(-x)}{D(-x)}=-\xi
$$

From (2.34), we have

$$
\xi=-\frac{1}{\alpha}=\sum_{k=1}^{\infty} \frac{1}{\mu_{k} \pi_{k}}
$$

which by (3.1) and (3.2) leads to (3.26).

Remark 3.3.4. The formula (3.26) can be established directly using twice the integral formula $[15,7.512(12)]$ which expresses ${ }_{p+1} F_{q+1}$ as an integral of ${ }_{p} F_{q}$. This relates the ${ }_{4} F_{3}$ to a special ${ }_{2} F_{1}$ which is known

$$
{ }_{2} F_{1}\left[\begin{array}{l}
1,1 \\
3 / 2
\end{array} ; x\right]=\frac{\operatorname{Arcsin} \sqrt{x}}{\sqrt{x(1-x)}} \quad 0 \leq x<1 .
$$

An interchange of the order of integration followed by an integration by parts leads to relation (3.26).

We do not know any formula which expresses $\xi$ in terms of classical constants. However, using the trigonometric series for cn $u$ (cf. $[15,8.146(2)])$ and integrating termwise, one gets

where

$$
\xi=\frac{K_{0}^{2}}{4}\left(\beta_{1}-\frac{2}{\pi^{2}} \beta_{3}\right)
$$

$$
\beta_{j}=\sum_{n=1}^{\infty} \frac{(-1)^{n-1}}{(n-1 / 2)^{j} \cosh (\pi(n-1 / 2))}, \quad j=1,3 .
$$

Here $\beta_{1}=\pi / 4$, cf. [23], but we do not know a similar simple evaluation of $\beta_{3}$.

By (2.24), we have

$$
\sum_{k=0}^{\infty} P_{k}^{2}(z)=B^{\prime}(z) D(z)-B(z) D^{\prime}(z), \quad z \in \mathbb{C}
$$


which can be reduced to

$$
\sum_{k=0}^{\infty} P_{k}^{2}(z)=\frac{K_{0}^{2}}{4 \pi}\left(\frac{\sin u}{u}+\frac{\sinh u}{u}+2 \frac{\sin u}{u} \frac{\sinh u}{u}\right), \quad u=z^{1 / 4} K_{0} .
$$

This gives, in particular, the function $1 / \rho(x)$ for $x \in \mathbb{R}$, cf. (2.14).

The identity (2.13) reduces to

$$
\delta_{0}(\tilde{z}) \Delta_{0}(\tilde{z})+\delta_{2}(\tilde{z}) \Delta_{2}(\tilde{z})=\frac{\pi}{4},
$$

a rather remarkable relation for which we now give a direct proof. To this end, we integrate $f(z) \mathrm{cn} z$ along the boundary of the rectangle with vertices $\pm K_{0}, \pm K_{0}+2 i K_{0}$, where $f$ is an arbitrary entire function. There is a simple pole at $z=i K_{0}$, and the residue theorem gives

$$
\begin{aligned}
\int_{-K_{0}}^{K_{0}}[f(x) & \left.+f\left(x+2 i K_{0}\right)+f\left(K_{0}+i\left(K_{0}+x\right)\right)+f\left(-K_{0}+i\left(K_{0}+x\right)\right)\right] \operatorname{cn} x d x \\
= & 2 \sqrt{2} \pi f\left(i K_{0}\right) .
\end{aligned}
$$

The choice of $f(z)=\exp \left(j p\left(z-i K_{0}\right)\right), p \in \mathbb{C}, j=\exp (i \pi / 4)$ and use of the relation (3.10) gives

$$
\int_{-K_{0}}^{K_{0}} \delta_{0}\left(p\left(x+i K_{0}\right)\right) \operatorname{cn} x d x=\frac{\pi}{\sqrt{2}} .
$$

By the addition formula (3.12) for $\delta_{0}$ and omitting zero contributions from odd functions, we find

$$
\frac{1}{\sqrt{2}} \int_{0}^{K_{0}}\left(\delta_{0}\left(p K_{0}\right) \delta_{0}(p x)+\delta_{2}\left(p K_{0}\right) \delta_{2}(p x)\right) \operatorname{cn} x d x=\frac{\pi}{4},
$$

which is (3.28) with $p=z^{1 / 4} / \sqrt{2}$.

3.4. The Nevanlinna parametrization. By Theorem 3.3.2, we have

$$
A(z)=\frac{1}{\sqrt{z}} \Delta_{2}(\tilde{z})-\xi C(z), \quad B(z)=-\delta_{0}(\tilde{z})-\xi D(z)
$$

so using the transformation

$$
\varphi^{\sharp}=-\frac{4}{\pi}\left(\xi+\frac{1}{\varphi}\right),
$$

we see that the Nevanlinna parametrization (2.3) takes the simple form

$$
\int \frac{d \nu_{\varphi}(u)}{z-u}=\frac{\frac{1}{\sqrt{z}} \Delta_{2}(\tilde{z})+\varphi^{\sharp}(z) \Delta_{0}(\tilde{z})}{-\delta_{0}(\tilde{z})+\varphi^{\sharp}(z) \sqrt{z} \delta_{2}(\tilde{z})},
$$

and the density $d(x)$ from Proposition 2.1.1 can be written

$$
d(x)=\frac{1}{4} \frac{\operatorname{Im} \varphi^{\sharp}\left(x^{+}\right)}{\left|\delta_{0}(\tilde{x})-\varphi^{\sharp}\left(x^{+}\right) \sqrt{x} \delta_{2}(\tilde{x})\right|^{2}},
$$

where $\sqrt{x}=i \sqrt{|x|}, \tilde{x}=j|x|^{1 / 4} K_{0} / \sqrt{2}$ if $x<0$. Note that (3.29) defines a homeomorphism of $\mathcal{P} \cup\{\infty\}$ for which the interval $\left[-\frac{1}{\xi}, 0\right]$ corresponds to $[0, \infty]$. 
The N-extremal solutions $\nu_{t}$ corresponding to $\varphi \equiv t \in \mathbb{R} \cup\{\infty\}$ are discrete measures concentrated at the zeros of the denominator in (3.30), i.e., the solution $z \in \mathbb{C}$ to the equation

$$
\sqrt{z} \frac{\delta_{2}(\tilde{z})}{\delta_{0}(\tilde{z})}=\frac{1}{t^{\sharp}}
$$

which by (3.10) and (3.11) are given by

$$
f(z)=\sqrt{z} \tan \left(z^{1 / 4} \frac{K_{0}}{2}\right) \tanh \left(z^{1 / 4} \frac{K_{0}}{2}\right)=\frac{1}{t^{\sharp}}=-\frac{\pi}{4} \frac{1}{\xi+\frac{1}{t}} .
$$

It is known from the general theory that (3.32) has only real solutions. There are two cases for which the equation can be solved explicitly, namely for $t=0,\left(t^{\sharp}=\infty\right)$ and $t=-1 / \xi\left(t^{\sharp}=0\right)$.

In the first case, the solutions are given by

$$
z_{n}=\left(\frac{2 n \pi}{K_{0}}\right)^{4}, \quad n=0,1, \ldots
$$

and the corresponding masses are

$$
\rho\left(z_{0}\right)=\frac{\pi}{K_{0}^{2}}, \rho\left(z_{n}\right)=\frac{4 \pi}{K_{0}^{2}} \frac{2 n \pi}{\sinh (2 n \pi)}, \quad n=1,2, \ldots,
$$

where $\rho$ is given by $(3.27)$.

In the second case, $t=-1 / \xi$, we find

$$
z_{n}=\left(\frac{(2 n+1) \pi}{K_{0}}\right)^{4}, \rho\left(z_{n}\right)=\frac{4 \pi}{K_{0}^{2}} \frac{(2 n+1) \pi}{\sinh ((2 n+1) \pi)}, \quad n=0,1, \ldots
$$

Summarizing, we have proved:

Proposition 3.4.1. The $N$-extremal measures $\nu_{0}$ and $\nu_{-1 / \xi}$ are given by

$$
\begin{gathered}
\nu_{0}=\frac{\pi}{K_{0}^{2}} \varepsilon_{x_{0}}+\frac{4 \pi}{K_{0}^{2}} \sum_{n=1}^{\infty} \frac{2 n \pi}{\sinh (2 n \pi)} \varepsilon_{x_{n}}, \quad x_{n}=\left(\frac{2 n \pi}{K_{0}}\right)^{4}, \\
\nu_{-1 / \xi}=\frac{4 \pi}{K_{0}^{2}} \sum_{n=0}^{\infty} \frac{(2 n+1) \pi}{\sinh ((2 n+1) \pi)} \varepsilon_{x_{n}}, \quad x_{n}=\left(\frac{(2 n+1) \pi}{K_{0}}\right)^{4} .
\end{gathered}
$$

Remark 3.4.2. The two measures above are in agreement with the measures derived in [28], but their $\mathrm{N}$-extremal character is now established. We note that it was proved by another method in [7] that $\nu_{0}$ is $\mathrm{N}$-extremal.

From Remark 2.2.2, we know that $\operatorname{supp}\left(\nu_{t}\right) \subseteq[0, \infty[$ for $t \in[-1 / \xi, 0]$, and for $t \notin[-1 / \xi, 0]$ the measure $\nu_{t}$ has one negative point in the support. This is in agreement with (3.32) because the function $f(z)$ is strictly increasing from $-\infty$ to $\infty$ in each of the intervals $]-\infty,\left(\pi / K_{0}\right)^{4}[,]\left((2 n-1) \pi / K_{0}\right)^{4},\left((2 n+1) \pi / K_{0}\right)^{4}[, n=1,2, \ldots$ For $z=-x, x>0$, one can express $f(z)$ as

$$
f(z)=\sqrt{x} \frac{\cos (\tilde{x})-\cosh (\tilde{x})}{\cos (\tilde{x})+\cosh (\tilde{x})}, \quad \tilde{x}=\frac{x^{1 / 4}}{\sqrt{2}} K_{0} .
$$


Remark 3.4.3. The mass of $\nu_{0}$ at $x_{n}=\left(2 n \pi / K_{0}\right)^{4}$ is also equal to the residue of (3.30) at $x_{n}$ which leads to

$$
\Delta_{0}\left(\frac{2 n \pi}{\sqrt{2}}\right)=\frac{\pi}{4} \frac{(-1)^{n}}{\cosh (n \pi)}, \quad n \geq 0
$$

Similarly, we find

$$
\Delta_{2}\left(\frac{(2 n+1) \pi}{\sqrt{2}}\right)=\frac{\pi}{4} \frac{(-1)^{n}}{\sinh \left(n+\frac{1}{2}\right) \pi}, \quad n \geq 0 .
$$

3.5. Examples of non-N-extremal measures. The simplest way to obtain examples of measures in $V$ which are not $\mathrm{N}$-extremal is to use the convexity of $V$. Starting from $\nu_{0}$ and $\nu_{-1 / \xi}$, we may take the convex combination with $s=(1-a) / 2$, $a \in[-1,1]$. This gives the one-parameter family

$$
\nu^{(a)}=\frac{1-a}{2} \nu_{-1 / \xi}+\frac{1+a}{2} \nu_{0}, \quad-1 \leq a \leq 1,
$$

and using the explicit form of these measures, we find

$$
\nu^{(a)}=\frac{(1+a)}{2 K_{0}^{2}} \pi \varepsilon_{x_{0}}+\frac{2 \pi^{2}}{K_{0}^{2}} \sum_{n=1}^{\infty} \frac{n}{\sinh (n \pi)}\left(1+a(-1)^{n}\right) \varepsilon_{x_{n}}
$$

with $x_{n}=\left(n \pi / K_{0}\right)^{4}, n=0,1, \ldots$

The corresponding function $\varphi_{a} \in \mathcal{P}$ follows from the relation (2.18) and is given by

$$
\frac{1}{\varphi_{a}(z)}+\xi=\frac{\pi}{4} \frac{1+a}{1-a} \frac{\delta_{0}(\tilde{z})}{\sqrt{z} \delta_{2}(\tilde{z})}
$$

For $a \neq \pm 1$, this gives a simple example of a non-N-extremal measure, which was first derived in [28] using a different approach.

From (2.17), we get an example of an absolutely continuous measure

$$
\nu_{\varphi_{t, \gamma}}=\frac{\gamma}{\pi} \frac{d x}{(t B(x)-D(x))^{2}+\gamma^{2} B(x)^{2}},
$$

with support equal to the whole real axis. Here $t \in \mathbb{R}$ and $\gamma>0$ are parameters. When $t$ and $\gamma$ are related by the equation

$$
\left.\gamma^{2}=-\frac{t}{\xi}-t^{2}, \quad t \in\right]-1 / \xi, 0[
$$

the parameter

$$
p=\frac{4}{\pi} \frac{\gamma}{t^{2}+\gamma^{2}}=\frac{4}{\pi} \xi \sqrt{-1-\frac{1}{t \xi}}
$$

can attain any value in $] 0, \infty[$, and the above density can be expressed

$$
\nu_{\varphi_{p}}=\frac{p}{4} \frac{d x}{\delta_{0}^{2}(\tilde{x})+p^{2} x \delta_{2}^{2}(\tilde{x})}, \quad \tilde{x}=\frac{x^{1 / 4}}{\sqrt{2}} K_{0} .
$$

The corresponding $\varphi_{p} \in \mathcal{P}$ is given by $\varphi_{p}(z)=t+i \gamma, \operatorname{Im}(z)>0$. 
Using (3.10) and (3.11), the denominator in (3.34) can be reduced to the following expression

$$
\begin{cases}\cos ^{2} u \cosh ^{2} u+p^{2}\left(\frac{2 u}{K_{0}}\right)^{4} \sin ^{2} u \sinh ^{2} u, & \text { for } x \geq 0 \\ \left(\frac{\cos u+\cosh u}{2}\right)^{2}+p^{2}\left(\frac{2 u}{K_{0}}\right)^{4}\left(\frac{\cos u-\cosh u}{2}\right)^{2}, & \text { for } x<0\end{cases}
$$

where $u=|x|^{1 / 4} K_{0} / 2$.

When $\varphi^{\sharp}(z)=-1 / \sqrt{z}$, then $\nu_{\varphi}$ has a discrete component with masses at the zeros of $\delta_{0}(\tilde{z})+\delta_{2}(\tilde{z})$, and an absolutely continuous component concentrated on $]-\infty, 0[$. The zeros are the numbers $z_{n}=\left(2 u_{n} / K_{0}\right)^{4}$, where $u_{n}$ are the solutions of

$$
\left.\tan u_{n} \tanh u_{n}=-1, \quad u_{n} \in\right](n-1 / 2) \pi, n \pi[, \quad n \geq 1 .
$$

The density $d(x)$ is easily calculated using (3.31), and we find

$$
d(x)=\frac{K_{0}^{2}}{4} \frac{1}{v^{2}\left(\cos ^{2} v+\cosh ^{2} v\right)}, \quad v=(-x)^{1 / 4} \frac{K_{0}}{\sqrt{2}}, \quad x<0 .
$$

To conclude let us present an example where both the absolutely continuous and discrete component can be given explicitly. We take

$$
\varphi^{\sharp}(z)=-\frac{K_{0}^{2} \gamma}{4} \cot \left(\frac{\tilde{z}}{\sqrt{2}}\right), \quad \gamma>0
$$

for which

$$
\int \frac{d \nu_{\varphi}(x)}{z-x}=\frac{\Delta_{2}(\tilde{z}) / \sqrt{z}-\frac{1}{4} K_{0}^{2} \gamma \Delta_{0}(\tilde{z}) \cot (\tilde{z} / \sqrt{2})}{-\cos (\tilde{z} / \sqrt{2})\left\{\cosh (\tilde{z} / \sqrt{2})+\gamma(\tilde{z} / \sqrt{2})^{2} \sinh (\tilde{z} / \sqrt{2})\right\}} .
$$

The denominator has zeros at $z_{n}^{(1)}=\left((2 n+1) \pi / K_{0}\right)^{4}, n \geq 0$. The residue of (3.37) at $z_{n}^{(1)}$ can be calculated using Remark 3.4.3, and putting $u_{n}=\left(n+\frac{1}{2}\right) \pi$, we find

$$
\alpha_{n}=\operatorname{Res}\left(z=z_{n}^{(1)}\right)=\frac{4 \pi}{K_{0}^{2}} \frac{u_{n}}{\sinh u_{n}\left(\cosh u_{n}+\gamma u_{n}^{2} \sinh u_{n}\right)} .
$$

The numerator has poles at $z_{n}^{(2)}=\left(2 n \pi / K_{0}\right)^{4}, n \geq 0$, and, as above, the residue of (3.37) at $z_{n}^{(2)}$ can be calculated, and putting $v_{n}=n \pi$, we find

$$
\beta_{n}=\operatorname{Res}\left(z=z_{n}^{(2)}\right)=\frac{4 \pi}{K_{0}^{2}} \frac{\gamma v_{n}^{3}}{\cosh v_{n}\left(\cosh v_{n}+\gamma v_{n}^{2} \sinh v_{n}\right)} .
$$

Note that $\beta_{0}=0$ so that there is in fact no pole at $z_{0}^{(2)}=0$.

To calculate the absolutely continuous part on the negative half-axis, we put

$$
v=(-x)^{1 / 4} \frac{K_{0}}{\sqrt{2}} \text { for } x<0
$$

and find the density

$$
h(x)=\frac{K_{0}^{2}}{4} \frac{\gamma \sinh v}{(\cosh v-\cos v)\left\{\left(\cos v+\cosh v-\frac{1}{2} \gamma v^{2} \sin v\right)^{2}+\left(\frac{1}{2} \gamma v^{2} \sinh v\right)^{2}\right\}} .
$$


To summarize, the measure $\nu_{\varphi}$ from (3.37) is given by

$$
\nu_{\varphi}=\sum_{n=0}^{\infty} \alpha_{n} \varepsilon_{z_{n}^{(1)}}+\sum_{n=0}^{\infty} \beta_{n} \varepsilon_{z_{n}^{(2)}}+1_{]-\infty, 0]}(x) h(x) d x .
$$

3.6. Asymptotic behaviour of the moments. Using the measure $\nu^{(a)}$ in (3.33) for $a=0$, we get the following formula for the moments

$$
s_{n}=\frac{\pi}{2 K_{0}^{2}} \delta_{n 0}+\frac{2 \pi^{2}}{K_{0}^{2}} \sum_{k=1}^{\infty} \frac{k}{\sinh (k \pi)}\left(\frac{k \pi}{K_{0}}\right)^{4 n}, \quad n=0,1, \ldots,
$$

and the fact that all the measures $\nu^{(a)}$ have the same moments leads to the equations

$$
\sum_{k=1}^{\infty} \frac{(-1)^{k} k^{4 n+1}}{\sinh (k \pi)}=-\frac{1}{4 \pi} \delta_{n 0}, \quad n=0,1, \ldots
$$

which have a long history, cf. [28]. Using (3.39), it was proved in [28, formula (49)] that we have the following generating function for the moments

$$
\varphi(x)=\frac{\operatorname{cn}(\sqrt{2} x)}{\operatorname{dn}^{2}(\sqrt{2} x)}=\sum_{n=0}^{\infty}(-1)^{n} s_{n} \frac{x^{4 n}}{(4 n) !} .
$$

The function $\varphi$ has double poles at the zeros of $\operatorname{dn}(\sqrt{2} x)$, i.e., at the points

$$
x=\frac{K_{0}}{\sqrt{2}}(2 n+1)+i \frac{K_{0}}{\sqrt{2}}(2 m+1), \quad n, m \in \mathbb{Z} .
$$

The radius of convergence of (3.41) is, therefore, $K_{0}$ and the Hadamard formula for the radius of convergence gives

$$
\limsup _{n \rightarrow \infty} \frac{\sqrt[4 n]{s_{n}}}{n}=\frac{4}{K_{0} e}
$$

By the Darboux method, we can obtain more precise information.

Proposition 3.6.1. The moments satisfy

$$
s_{n}=\frac{4(4 n+1) !}{K_{0}^{4 n+2}}\left(1+O\left(\frac{1}{25^{n}}\right)\right) .
$$

Proof. Putting $j=\exp \left(i \frac{\pi}{4}\right)$, we have

$$
\varphi\left(j K_{0}+x\right)=2 i \frac{\mathrm{cn}(\sqrt{2} x)}{\operatorname{sn}^{2}(\sqrt{2} x)}
$$

and using

$$
\frac{\mathrm{cn} x}{\operatorname{sn}^{2} x}=\frac{1}{x^{2}}-\frac{3}{40} x^{2}+\cdots
$$

in a neighbourhood of zero, the principal part of $\varphi$ corresponding to the pole $x=j K_{0}$ is $i\left(x-j K_{0}\right)^{-2}$. The principal part corresponding to the remaining poles $-j K_{0}, \pm \bar{j} K_{0}$ can be found in a similar way, and the sum $s(x)$ of the four principal parts has the following power series expansion for $|x|<K_{0}$

$$
\sum_{n=0}^{\infty}(-1)^{n} \frac{4(4 n+1)}{K_{0}^{4 n+2}} x^{4 n} \text {. }
$$


The function $\varphi(x)-s(x)$ is holomorphic for $|x|<\sqrt{5} K_{0}$ with 8 double poles on the circle $|x|=\sqrt{5} K_{0}$, and therefore we get

$$
(-1)^{n} \frac{s_{n}}{(4 n) !}=(-1)^{n} \frac{4(4 n+1)}{K_{0}^{4 n+2}}+O\left(\frac{n}{\left(\sqrt{5} K_{0}\right)^{4 n}}\right),
$$

which proves the assertion.

\section{Al-Salam-Carlitz $q$-polynomials}

These polynomials, $V_{n}=V_{n}^{(a)}(x ; q)$, were introduced in [2]. They are connected to the birth and death process with rates

$$
\lambda_{n}=a q^{-n} ; \quad \mu_{n}=q^{-n}-1, \quad n \geq 0,
$$

by the formula

$$
F_{n}(x)=\sigma_{n} V_{n}(1+x) ; \quad \sigma_{n}=(-1)^{n} \frac{q^{n(n+1) / 2}}{[q]_{n}}
$$

cf. [7], where $F_{n}=F_{n}^{(a)}(x ; q)$ are defined by $(2.28),(2.29)$. We always restrict the parameters $a, q$ to the domain

$$
\Omega=\{(a, q) \mid a>0,0<q<1\}
$$

for which $\lambda_{n}>0, \mu_{n+1}>0$ when $n \geq 0$ and $\mu_{0}=0$. Since we only consider one fixed value of $q$, we use the simplified notation $[z]_{n}$ instead of $(z ; q)_{n}$, i.e.,

$$
[z]_{n}=(z ; q)_{n}=\prod_{j=1}^{n}\left(1-z q^{j-1}\right), \quad z \in \mathbb{C}, n=0,1, \ldots, \infty
$$

(with $\left.[z]_{0}=1\right)$. By $(2.30)$, we get

$$
\pi_{n}=\pi_{n}^{(a)}(q)=\frac{(a q)^{n}}{[q]_{n}} ; \quad \lambda_{n-1} \pi_{n-1}=\frac{a^{n}}{[q]_{n-1}}
$$

and, concerning determinacy, we have, cf. [11], [7]:

$$
\begin{gathered}
\operatorname{indet}(H) \Leftrightarrow q<a<q^{-1}, \\
\operatorname{indet}(S) \Leftrightarrow 1<a<q^{-1}, \\
\operatorname{indet}(H), \operatorname{det}(S) \Leftrightarrow q<a \leq 1 .
\end{gathered}
$$

Al-Salam and Carlitz found the following discrete solution of the corresponding moment problem

$$
\beta^{(a)}=K \sum_{n=0}^{\infty} \frac{a^{n} q^{n^{2}}}{[a q]_{n}[q]_{n}} \varepsilon_{\left(q^{-n}-1\right)},
$$

which is a positive measure for $a q<1$. The constant $K$ was evaluated later by Ismail [16] who found $K=[a q]_{\infty}$. Below, we shall deduce this once more as well as finding orthogonality measures for the remaining parameter values. 


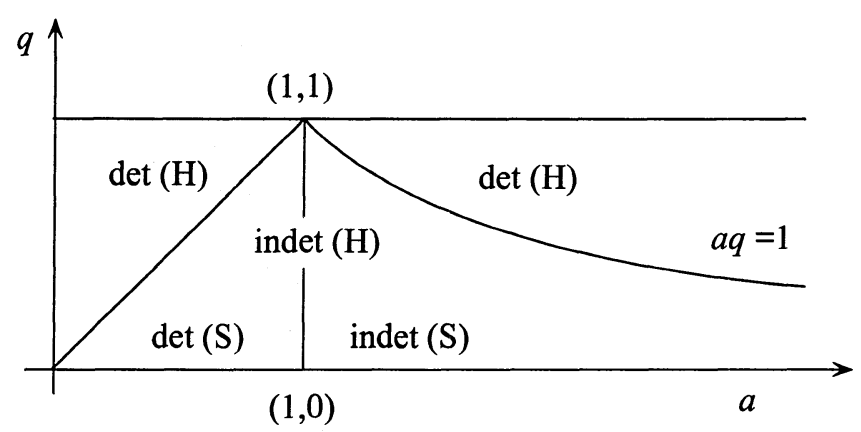

Figure 1. Domains of determinacy/indeterminacy

4.1. Invariance properties under the transformation $a \mapsto 1 / a$. Dividing the equation $(2.28)$ by $a^{n+1}$ and using the fact that $F_{n}^{(a)}(x ; q)$ is uniquely determined by (2.28) together with the initial conditions (2.29), we find

$$
F_{n}^{(1 / a)}(x ; q)=a^{-n} F_{n}^{(a)}((1+x) a-1 ; q), \quad(a, q) \in \Omega ; x \in \mathbb{C} .
$$

The same equation holds for $\tilde{F}_{n}$.

Using (2.32), we get the following invariance for the corresponding orthonormal polynomials $P_{n}^{(a)}(x ; q)$ and the polynomials $Q_{n}^{(a)}(x ; q)$ of the second kind,

$$
\left.\begin{array}{l}
P_{n}^{(1 / a)}(x ; q)=P_{n}^{(a)}((1+x) a-1 ; q) \\
Q_{n}^{(1 / a)}(x ; q)=a Q_{n}^{(a)}((1+x) a-1 ; q)
\end{array}\right\}, \quad(a, q) \in \Omega, x \in \mathbb{C} .
$$

The domain

$$
\Gamma=\left\{(a, q) \in \Omega \mid q<a<\frac{1}{q}\right\}
$$

of indeterminacy in the sense of Hamburger is invariant under $a \mapsto 1 / a$. We shall next show that the assumptions of Proposition 2.4.1 are verified for $\Gamma$ and shall examine the invariance of the entire functions $\mathcal{A}^{(a)}(z, w ; q), \ldots$ from $(2.10)$. In the following, we omit $q$ in the notation.

Proposition 4.1.1. The functions $\mathcal{A}, \mathcal{B}, \mathcal{D}$ from (2.10) are continuous on $\mathbb{C}^{2} \times \Gamma$ and have the invariance properties

$$
\begin{gathered}
\mathcal{A}^{(1 / a)}(z, w)=a \mathcal{A}^{(a)}((1+z) a-1,(1+w) a-1) \\
\mathcal{B}^{(1 / a)}(z, w)=\mathcal{B}^{(a)}((1+z) a-1,(1+w) a-1) \\
\mathcal{D}^{(1 / a)}(z, w)=\frac{1}{a} \mathcal{D}^{(a)}((1+z) a-1,(1+w) a-1),
\end{gathered}
$$

for $(z, w) \in \mathbb{C}^{2},(a, q) \in \Gamma$.

Proof. Since the rates $\lambda_{n}, \mu_{n}$ depend ntinuously on $(a, q)$, we only have to verify the uniform convergence of the series in (2.36) on a fixed compact subset $\Gamma_{0} \subseteq \Gamma$, cf. Remark 2.4.2, and for this it suffices to find convergent majorant series.

We have

$$
\left(P_{n}^{(a)}(0 ; q)\right)^{2}=\pi_{n}^{(a)}(q)=\frac{(a q)^{n}}{[q]_{n}} \leq \frac{r^{n}}{[q]_{n}}
$$


where $r=\max _{\Gamma_{0}}(a q)<1$, which settles the question for the first series. Putting $\max _{\Gamma_{0}}(q)=q_{0}, \max _{\Gamma_{0}}(q / a)=s$, we have $q_{0}<1, s<1$, and

$$
\left(Q_{n}^{(a)}(0 ; q)\right)^{2}=\frac{(a q)^{n}}{[q]_{n}}\left(\sum_{k=1}^{n} \frac{[q]_{k-1}}{a^{k}}\right)^{2} \leq n^{2} \frac{(\max (r, s))^{n}}{\left[q_{0}\right]_{n}}
$$

where we use

$$
\sum_{k=1}^{n} \frac{[q]_{k-1}}{a^{k}} \leq \begin{cases}n, & \text { if } a \geq 1 \\ \frac{n}{a^{n}}, & \text { if } a<1\end{cases}
$$

The invariance formulas for $\mathcal{A}, \mathcal{B}$, and $\mathcal{D}$ are easy consequences of (4.8) and (2.10).

For the explicit determination of the functions $\mathcal{A}, \mathcal{B}, \mathcal{D}$, it will suffice to carry through the calculations for $a<1$ and then use the above properties.

4.2. The generating functions. The starting point of the asymptotic analysis in the indeterminate case is the two generating functions

$$
\begin{gathered}
F(z, w)=\sum_{n=0}^{\infty} F_{n}(z) w^{n}=\frac{[(1+z) q w]_{\infty}}{[q w]_{\infty}[a q w]_{\infty}} \\
\frac{1}{\mu_{1}} \tilde{F}(z, w)=\sum_{n=0}^{\infty} \frac{\tilde{F}_{n}(z)}{\mu_{1}} w^{n} \\
=\frac{q}{(1-q w)(1-a q w)}{ }_{3} \phi_{2}\left[\begin{array}{c}
(1+z) q w, q, 0 \\
q^{2} w, a q^{2} w
\end{array} ; q\right]
\end{gathered}
$$

valid for $z \in \mathbb{C},|w| \leq 1$ when the moment problem is indet $(H)$, cf. [7]. Here ${ }_{3} \phi_{2}$ is the basic hypergeometric series as defined in [14] and evaluated at $q$. The basic $q$ is omitted in the notation.

The right-hand sides of (4.10) and (4.11) are meromorphic functions of $w$ for each $z \in \mathbb{C}$ with poles

$$
w_{n}^{(1)}=\frac{1}{q^{n}}, \quad w_{n}^{(2)}=\frac{1}{a q^{n}}, \quad n=1,2, \ldots
$$

Reasoning backwards, one can verify that the power series expansions (4.10), (4.11) in $w$ of these functions are given by polynomial coefficients $F_{n}(z),\left(1 / \mu_{1}\right) \tilde{F}_{n}(z)$ satisfying (2.28), (2.29). We therefore conclude that (4.10) and (4.11) hold for $(a, q) \in \Omega, z \in \mathbb{C}$ and $|w|<R$, where $R$ is the distance from the origin to the closest pole.

We remark in passing that the generating functions $F, \tilde{F}$ both have the following invariance property

$$
f^{(1 / a)}(z, a w)=f^{(a)}((1+z) a-1, w)
$$

where we have omitted $q$ in the notation. 
4.3. Asymptotic analysis. We restrict the calculation to the case $0<q<a<1$. We note that the poles (4.12) are simple in this case and verify

$$
\frac{1}{q}<\frac{1}{a q}<\frac{1}{q^{2}}<\frac{1}{a q^{2}}<\cdots
$$

The principal parts of $F(z, w)$ corresponding to the poles $w=1 / q$ and $w=1 /(a q)$ are

$$
\frac{[1+z]_{\infty}}{[a]_{\infty}[q]_{\infty}(1-q w)}, \quad \frac{[(1+z) / a]_{\infty}}{[1 / a]_{\infty}[q]_{\infty}(1-a q w)}
$$

respectively, and it follows that $F(z, w)$ minus these expressions is holomorphic for $|w|<1 / q^{2}$. By (4.10), this gives

$$
F_{n}(z)=\frac{q^{n}}{[q]_{\infty}} \frac{[1+z]_{\infty}}{[a]_{\infty}}+\frac{(a q)^{n}}{[q]_{\infty}} \frac{[(1+z) / a]_{\infty}}{[1 / a]_{\infty}}+O\left(q^{2 n}\right)
$$

The principal parts of $\left(1 / \mu_{1}\right) \tilde{F}(z, w)$ corresponding to the poles $w=1 / q$ and $w=$ $1 /(a q)$ are

$$
\frac{q}{(1-a)(1-q w)}{ }_{2} \phi_{1}\left[\begin{array}{c}
1+z, 0 \\
a q
\end{array} ; q\right], \quad \frac{q}{(1-1 / a)(1-a q w)}{ }_{2} \phi_{1}\left[\begin{array}{c}
(1+z) / a, 0 \\
q / a
\end{array} ; q\right],
$$

respectively, from which we deduce

$$
\frac{1}{\mu_{1}} \tilde{F}_{n-1}(z)=\frac{q^{n}}{1-a}{ }_{2} \phi_{1}\left[\begin{array}{c}
1+z, 0 \\
a q
\end{array} ; q\right]-\frac{(a q)^{n}}{1-a}{ }_{2} \phi_{1}\left[\begin{array}{c}
(1+z) / a, 0 \\
q / a
\end{array} ; q\right]+O\left(q^{2 n}\right) .
$$

Plugging (4.5), (4.14), and (4.15) in (2.35), we get after some calculation the following formulas:

Proposition 4.3.1. The entire functions $\mathcal{A}, \mathcal{B}, \mathcal{D}$ from (2.10) are given by

$$
\begin{gathered}
\mathcal{A}^{(a)}(z, w)=\frac{[q]_{\infty}}{1-a}\left\{{ }_{2} \phi_{1}\left[\begin{array}{c}
1+z, 0 \\
a q]
\end{array}\right]{ }_{2} \phi_{1}\left[\begin{array}{c}
(1+w) / a, 0 \\
q / a
\end{array} ; q\right]\right. \\
\left.-{ }_{2} \phi_{1}\left[\begin{array}{c}
(1+z) / a, 0 \\
q / a
\end{array} ; q\right]{ }_{2} \phi_{1}\left[\begin{array}{c}
1+w, 0 \\
a q
\end{array} ; q\right]\right\} \\
\mathcal{B}^{(a)}(z, w)=\frac{1}{1-a}\left\{\frac{a[(1+z) / a]_{\infty}}{[q / a]_{\infty}} \phi_{1}\left[\begin{array}{c}
1+w, 0 \\
a q
\end{array} ; q\right]\right. \\
\left.-\frac{[1+z]_{\infty}}{[q a]_{\infty}}{ }_{2} \phi_{1}\left[\begin{array}{c}
(1+w) / a, 0 \\
q / a
\end{array}\right]\right\} \\
\mathcal{D}^{(a)}(z, w)=\frac{a}{(1-a)[q]_{\infty}[a q]_{\infty}[q / a]_{\infty}}\left\{\left[\begin{array}{c}
{[1+z]_{\infty}[(1+w) / a]_{\infty}} \\
\left.-[(1+z) / a]_{\infty}[1+w]_{\infty}\right\}
\end{array}\right.\right.
\end{gathered}
$$

for $(z, w) \in \mathbb{C}^{2}$ and $(a, q) \in \Gamma, a \neq 1$. 
Remark 4.3.2. In the first place, we get the formulas only for $a<1$, but noting that the right-hand sides in the formulas have the same invariance properties as $\mathcal{A}, \mathcal{B}, \mathcal{D}$ in Proposition 4.1.1, we conclude that the above formulas hold for $(a, q) \in \Gamma, a \neq 1$. Since we know that $\mathcal{A}, \mathcal{B}, \mathcal{D}$ are continuous functions of the parameters, we find $\mathcal{A}^{(1)}$, $\mathcal{B}^{(1)}, \mathcal{D}^{(1)}$ by taking the limit of the above expressions for $a \rightarrow 1$. This leads to quite complicated expressions, so we shall not give the formulas.

The identities $(2.8)$ or $(2.13)$ reduce to

$$
\frac{[1+z]_{\infty}}{[a]_{\infty}}{ }_{2} \phi_{1}\left[\begin{array}{c}
(1+z) / a, 0 \\
q / a
\end{array} ; q\right]+\frac{[(1+z) / a]_{\infty}}{[1 / a]_{\infty}}{ }_{2} \phi_{1}\left[\begin{array}{c}
1+z, 0 \\
a q
\end{array} ; q\right]=1
$$

which is a particular case of (2.10.13) in [14].

\subsection{The Nevanlinna matrix.}

Theorem 4.4.1. For $(a, q) \in \Gamma, a \neq 1$, the Nevanlinna matrix consists of the following entire functions

$$
\begin{aligned}
& A^{(a)}(z)=\frac{[q]_{\infty}}{a-1}\left\{{ }_{2} \phi_{1}\left[\begin{array}{c}
(1+z) / a, 0 \\
q / a
\end{array} ; q\right]-{ }_{2} \phi_{1}\left[\begin{array}{c}
1 / a, 0 \\
q / a
\end{array} ; q\right]{ }_{2} \phi_{1}\left[\begin{array}{c}
1+z, 0 \\
a q
\end{array} ; q\right]\right\} \\
& B^{(a)}(z)=\frac{1}{a-1}\left\{\frac{[1+z]_{\infty}}{[q a]_{\infty}}{ }_{2} \phi_{1}\left[\begin{array}{c}
1 / a, 0 \\
q / a
\end{array} ; q\right]-\frac{a[(1+z) / a]_{\infty}}{[q / a]_{\infty}}\right\} \\
& C^{(a)}(z)={ }_{2} \phi_{1}\left[\begin{array}{cc}
1+z, 0 & ; q \\
a q &
\end{array}\right] \\
& D^{(a)}(z)=-\frac{[1+z]_{\infty}}{[q]_{\infty}[a q]_{\infty}} \text {. }
\end{aligned}
$$

They are all of order 0 . The functions $A^{(1)}(z), \ldots, D^{(1)}(z)$ are obtained for $a \rightarrow 1$.

Proof. This follows immediately from Proposition 4.3.1 and (2.12). Since they all have the same order, it suffices to determine the order of $D^{(a)}$, which is clearly 0 , since the zeros $z_{n}=q^{-n}-1$ have exponent of convergence equal to zero.

Defining the constant

$$
\xi(a)=\frac{[q]_{\infty}}{a-1}{ }_{2} \phi_{1}\left[\begin{array}{c}
1 / a, 0 \\
q / a
\end{array} ; q\right] \text { for } q<a<\frac{1}{q}, a \neq 1,
$$

we see that

$$
\begin{gathered}
A^{(a)}(z)=\frac{[q]_{\infty}}{a-1}{ }_{2} \phi_{1}\left[\begin{array}{c}
(1+z) / a, 0 \\
q / a
\end{array} ; q\right]-\xi(a) C^{(a)}(z), \\
B^{(a)}(z)=-\frac{a}{a-1} \frac{[(1+z) / a]_{\infty}}{[q / a]_{\infty}}-\xi(a) D^{(a)}(z),
\end{gathered}
$$

which is similar in structure to the Nevanlinna matrix in the quartic case. The expression for $\xi(a)$ can be simplified to

$$
\left.\xi(a)=[q]_{\infty} \sum_{n=0}^{\infty} \frac{q^{n}}{\left(a-q^{n}\right)[q]_{n}} \text { for } a \in\right] q, 1 / q[\backslash\{1\}
$$


Lemma 4.4.2. The function $\xi$ is strictly decreasing in each of the intervals $] q, 1[$, ] $1,1 / q[$ with

$$
\xi(q+)=\infty, \quad \xi(1-)=-\infty, \quad \xi(1+)=\infty, \quad \xi(1 / q-)=1-[q]_{\infty} .
$$

For $1<a<1 / q$, in which case the problem is $\operatorname{indet}(S)$, the constant $\alpha=\alpha(a)$ from (2.25) is given by

$$
\alpha(a)=-\frac{1}{\xi(a)}
$$

Proof. Formula (4.18) shows that $\xi$ is strictly decreasing with the above limits at $a=q, 1$ and

$$
\xi(1 / q-)=[q]_{\infty} \sum_{n=0}^{\infty} \frac{q^{n+1}}{[q]_{n+1}}=1-[q]_{\infty}
$$

by the $q$-binomial Theorem.

For $1<a<1 / q$, we have by (2.34),

$$
-\frac{1}{\alpha}=\sum_{n=0}^{\infty} \frac{[q]_{n}}{a^{n+1}}=\frac{1}{a}{ }_{2} \phi_{1}\left[\begin{array}{c}
q, q \\
0
\end{array} ; 1 / a\right]
$$

and using Heine's transformation for ${ }_{2} \phi_{1}$ (see [14, (1.4.1)]), we get $-1 / \alpha=\xi(a)$.

Another quantity of interest is the function $\rho=\rho^{(a)}(\cdot ; q)$ given by

$$
1 / \rho(z)=\sum_{n=0}^{\infty}\left|P_{n}(z)\right|^{2}, \quad z \in \mathbb{C}
$$

cf. (2.14). Differentiating $\mathcal{D}^{(a)}(z, w)$ with respect to $z$ and setting $z=w=x$ gives $1 / \rho(x)$ for $x \in \mathbb{R}$, cf. (2.10). Using

$$
h_{\infty}(z):=[z]_{\infty}^{\prime}=-[z q]_{\infty 2} \phi_{1}\left[\begin{array}{c}
z, q \\
z q
\end{array} ; q\right]
$$

we find for $a \neq 1, x \in \mathbb{R}$

$$
\begin{aligned}
\frac{1}{\rho(x)}=\frac{a[q(1+x)]_{\infty}[q(1+x) / a]_{\infty}}{(a-1)[q]_{\infty}[q a]_{\infty}[q / a]_{\infty}}\{(1- & \left.\frac{1+x}{a}\right){ }_{2} \phi_{1}\left[\begin{array}{c}
1+x, q \\
q(1+x)
\end{array} ; q\right] \\
+ & \left.\frac{x}{a}{ }_{2} \phi_{1}\left[\begin{array}{c}
(1+x) / a, q \\
q(1+x) / a
\end{array} ; q\right]\right\} .
\end{aligned}
$$

A formula valid for $x \in \mathbb{C}$ can be obtained using the generating function (1.17) in $[2]$

$$
\sum_{n=0}^{\infty} \Phi_{n}^{(a)}(z) \Phi_{n}^{(b)}(w) \frac{\zeta^{n}}{[q]_{n}}=\frac{[a z \zeta]_{\infty}[b w \zeta]_{\infty}}{[\zeta]_{\infty}[z \zeta]_{\infty}[w \zeta]_{\infty}}{ }_{3} \phi_{2}\left[\begin{array}{c}
a, b, \zeta \\
a z \zeta, b w \zeta
\end{array} ; z w \zeta\right]
$$

Here $\Phi_{n}^{(a)}$ is related to $V_{n}^{(a)}$ by the formula

$$
V_{n}^{(a)}(x)=(-1)^{n} q^{-\frac{1}{2} n(n-1)} \Phi_{n}^{(x / a)}(a)
$$

(which is (4.7) in [2], corrected), and replacing $a$ by $z / a, b$ by $w / b, z$ by $a, w$ by $b$, we get

$$
\sum_{n=0}^{\infty} q^{n(n-1)} V_{n}^{(a)}(z) V_{n}^{(b)}(w) \frac{\zeta^{n}}{[q]_{n}}=\frac{[z \zeta]_{\infty}[w \zeta]_{\infty}}{[\zeta]_{\infty}[a \zeta]_{\infty}[b \zeta]_{\infty}}{ }_{3} \phi_{2}\left[\begin{array}{c}
z / a, w / b, \zeta \\
z \zeta, w \zeta
\end{array} ; a b \zeta\right]
$$


Finally, for $a=b, \zeta=q / a$, we get by (4.2), (4.5), and (2.32)

$$
\begin{aligned}
\sum_{n=0}^{\infty} P_{n}^{(a)}(z) P_{n}^{(a)}(w)=\frac{[q(1+z) / a]_{\infty}[q(1+w) / a]_{\infty}}{[q / a]_{\infty}[q]_{\infty}^{2}} \\
\times{ }_{3} \phi_{2}\left[\begin{array}{c}
(1+z) / a,(1+w) / a, q / a \\
q(1+z) / a, q(1+w) / a
\end{array} ; a q\right]
\end{aligned}
$$

For $w=\bar{z}$, we get for $(a, q) \in \Gamma, z \in \mathbb{C}$ :

$$
\frac{1}{\rho^{(a)}(z)}=\frac{\left|[q(1+z) / a]_{\infty}\right|^{2}}{[q / a]_{\infty}[q]_{\infty}^{2}}{ }_{3} \phi_{2}\left[\begin{array}{c}
(1+z) / a,(1+\bar{z}) / a, q / a \\
q(1+z) / a, q(1+\bar{z}) / a
\end{array} ; q a\right] .
$$

The function $\rho(z)=\rho^{(a)}(z ; q)$ has the invariance property

$$
\rho^{(1 / a)}\left(\frac{1+z}{a}-1\right)=\rho^{(a)}(z)
$$

which follows immediately from (4.8), and this permits the transformation of (4.22) to the following

$$
\frac{1}{\rho^{(a)}(z)}=\frac{\left|[q(1+z)]_{\infty}\right|^{2}}{[q a]_{\infty}[q]_{\infty}^{2}}{ }_{3} \phi_{2}\left[\begin{array}{c}
1+z, 1+\bar{z}, q a \\
q(1+z), q(1+\bar{z})
\end{array} ; q / a\right],
$$

which will be useful later on.

4.5. The Nevanlinna parametrization. If we introduce the transformation analogous to (3.29)

$$
\varphi^{\#}=-\left(\xi(a)+\frac{1}{\varphi}\right)
$$

and use (4.17), we see that the Nevanlinna parametrization takes the form

$$
\int \frac{d \nu_{\varphi}^{(a)}(u)}{z-u}=\frac{\frac{[q]_{\infty}}{a-1}{ }_{2} \phi_{1}\left[\begin{array}{c}
(1+z) / a, 0 \\
q / a
\end{array} ; q\right]+\varphi^{\#}(z){ }_{2} \phi_{1}\left[\begin{array}{c}
1+z, 0 \\
a q
\end{array} ; q\right]}{-\frac{a}{a-1} \frac{[(1+z) / a]_{\infty}}{[q / a]_{\infty}}-\varphi^{\#}(z) \frac{[1+z]_{\infty}}{[q]_{\infty}[a q]_{\infty}}} .
$$

The N-extremal measures $\nu_{t}^{(a)}, t \in \mathbb{R} \cup\{\infty\}$ have mass-points at the zeros of the denominator. As in the quartic case, only for two particular values of $t$ is it possible to find the zeros explicitly.

For $t=0$, it is the zeros of $[1+z]_{\infty}$ which are $z_{n}=q^{-n}-1, n \geq 0$. The masses $m_{n}^{(a)}$ are given by the residues at $z_{n}$, and for the derivative $h_{\infty}(z)$ of $[z]_{\infty}$, it is easy to calculate

$$
h_{\infty}\left(q^{-n}\right)=(-1)^{n+1} q^{-n(n-1) / 2}[q]_{\infty}[q]_{n}, \quad n \geq 0
$$

Using

$$
{ }_{2} \phi_{1}\left[\begin{array}{c}
q^{-n}, 0 \\
c
\end{array} ; q\right]=(-1)^{n} \frac{c^{n} q^{\frac{1}{2} n(n-1)}}{[c]_{n}}
$$


which follows from (1.5.3) in [14] by taking the limit $b \rightarrow 0$, we find

$$
m_{n}^{(a)}=[a q]_{\infty} \frac{a^{n} q^{n^{2}}}{[a q]_{n}[q]_{n}}
$$

in agreement with [7], [2].

For $t=-1 / \xi(a)$, the zeros of the denominator in (4.26) are determined by $[(1+z) / a]_{\infty}=0$ so they are $z_{n}=a q^{-n}-1, n \geq 0$, and the corresponding masses $\sigma_{n}^{(a)}$ can be calculated as residues using (4.27) and (4.28), and we find

$$
\sigma_{n}^{(a)}=[q / a]_{\infty} \frac{a^{-n} q^{n^{2}}}{[q / a]_{n}[q]_{n}}
$$

Proposition 4.5.1. For $(a, q) \in \Gamma, a \neq 1$, we have the following $N$-extremal measures

$$
\begin{gathered}
\nu_{0}^{(a)}=\sum_{n=0}^{\infty} m_{n}^{(a)} \varepsilon_{\left(q^{-n}-1\right)}, \\
\nu_{-1 / \xi(a)}^{(a)}=\sum_{n=0}^{\infty} \sigma_{n}^{(a)} \varepsilon_{\left(a q^{-n}-1\right)} .
\end{gathered}
$$

For $1<a<1 / q$, in case of which the problem is $\operatorname{indet}(S)$, both measures are concentrated on $[0, \infty[$.

For $q<a<1$, in which case the problem is $\operatorname{det}(S)$, the measure $\nu_{0}^{(a)}$ is the unique solution to the moment problem supported by $\left[0, \infty\left[\right.\right.$, and the measure $\nu_{-1 / \xi(a)}^{(a)}$ has one negative point $a-1$ in the support.

In the case $a=1$, which is $\operatorname{det}(S)$, the unique measure supported by $[0, \infty[$ is

$$
\nu_{0}^{(1)}=\sum[q]_{\infty} \frac{q^{n^{2}}}{[q]_{n}^{2}} \varepsilon_{\left(q^{-n}-1\right)} .
$$

Remark 4.5.2. For the last part of the proposition, we use Proposition 2.4.1 to conclude that

$$
\lim _{a \rightarrow 1} \nu_{0}^{(a)}=\lim _{a \rightarrow 1} \nu_{-1 / \xi(a)}^{(a)}=\nu_{0}^{(1)} \quad \text { weakly. }
$$

The fact that $\nu_{0}^{(a)}$ and $\nu_{-1 / \xi(a)}^{(a)}$ are probability measures leads to the equation

$$
\sum_{n=0}^{\infty} \frac{a^{n} q^{n^{2}}}{[a q]_{n}[q]_{n}}=\frac{1}{[a q]_{\infty}}
$$

in the first place for $q<a<1 / q$, but by holomorphic continuation (4.31) is valid for $|a|<1 / q$.

Remark 4.5.3. The jumps of the N-extremal measure $\nu_{0}^{(a)}$ can also be found departing from (4.24). Using the definition of ${ }_{3} \phi_{2}$, we get

$$
\frac{1}{\rho^{(a)}(z)}=\frac{1}{[a q]_{\infty}[q]_{\infty}^{2}} \sum_{k=0}^{\infty}\left(\frac{q}{a}\right)^{k} \frac{[a q]_{k}}{[q]_{k}}\left|[1+z]_{k}\left[(1+z) q^{k+1}\right]_{\infty}\right|^{2}
$$

so for $z_{n}=q^{-n}-1, n \geq 0$, all terms in the series vanish except for $k \neq n$, and we get

$$
\frac{1}{\rho^{(a)}\left(z_{n}\right)}=\frac{[q]_{n}[a q]_{n}}{a^{n} q^{n^{2}}[a q]_{\infty}}, \quad n \geq 0,
$$


in agreement with (4.29). Similarly, the jumps of $\nu_{-1 / \xi(a)}^{(a,}$ can be found using (4.22).

4.6. Examples of non-N-extremal measures. The simplest explicit examples of this type are obtained for $a \neq 1$ by taking convex combinations of $\nu_{0}^{(a)}$ and $\nu_{-1 / \xi(a)}^{(a)}$. The corresponding Pick functions are given by (2.18). In particular, we get

$$
\frac{[(1+z) / a]_{\infty}}{[1+z]_{\infty}} \in \mathcal{P} \quad \text { for } 1 \leq a \leq \frac{1}{q}, \quad \frac{[1+z]_{\infty}}{[(1+z) / a]_{\infty}} \in \mathcal{P} \quad \text { for } q \leq a \leq 1,
$$

so we have found the following examples of Pick functions $[z \lambda]_{\infty} /[z]_{\infty},[z]_{\infty} /[z / \lambda]_{\infty}$ for $q \leq \lambda \leq 1$.

The Pick function $\varphi_{t, \gamma}$ given by (2.16) leads to the absolutely continuous measure (2.17). As in the quartic case, we get a simpler expression if we assume that $\gamma>0$ and $\xi(a)$ are related by $\gamma^{2}=-t(1 / \xi(a)+t)$, where $t$ belongs to the interval with endpoints 0 and $-1 / \xi(a)$. The parameter $p=\gamma /\left(t^{2}+\gamma^{2}\right)$ can attain any value in $] 0, \infty[$ and the corresponding Pick function $\varphi_{p}$ leads to the measure

$$
\nu_{\varphi_{p}}=\frac{p}{\pi}\left\{\left(\frac{a}{a-1} \frac{[(1+x) / a]_{\infty}}{[q / a]_{\infty}}\right)^{2}+p^{2}\left(\frac{[1+x]_{\infty}}{[q]_{\infty}[a q]_{\infty}}\right)^{2}\right\}^{-1} d x .
$$

This leads to the following

Proposition 4.6.1. For $q<a<1 / q, a \neq 1$, we have the following one-parameter family of analytic densities for the corresponding moment problem

$$
\nu(x ; a, \rho)=c(a) \frac{\rho}{[(1+x) / a]_{\infty}^{2}+\rho^{2}[1+x]_{\infty}^{2}}, \quad 0<\rho<\infty
$$

where

$$
c(a)=\frac{|a-1|}{\pi a}[q]_{\infty}[a q]_{\infty}[q / a]_{\infty} .
$$

Remark 4.6.2. For $a \rightarrow 1$ and $\rho>0$ fixed, the measures $\nu(x ; a, \rho) d x$ converge weakly to $\nu_{0}^{(1)}$ from Proposition 4.5.1. In fact, since $|\xi(a)| \rightarrow \infty$ by Lemma 4.4.2, the parameters $t$ and $\gamma$ tend to zero, so the Pick function corresponding to $\nu(x ; a, \rho) d x$ tends to 0 in $\mathcal{P}$, and we can apply Proposition 2.4.1.

4.7. The case $a=1$. We shall give explicit formulas for the functions in the Nevanlinna matrix when $a=1$. The formulas are obtained from Theorem 4.4.1 by letting $a \rightarrow 1$. The calculations of $A^{(1)}$ and $B^{(1)}$ are obtained by differentiation with the respect to $a$, and therefore it is convenient to introduce the functions

$$
h_{n}(z)=[z]_{n}^{\prime}=-g_{n}(z)[z]_{n}, \quad n=0,1, \ldots, \infty
$$

with

$$
g_{n}(z)=\sum_{j=0}^{n-1} \frac{q^{j}}{1-z q^{j}}
$$

Note that

$$
g_{\infty}(z)=\sum_{j=0}^{\infty} \frac{q^{j}}{1-z q^{j}}=\frac{1}{1-z}{ }_{2} \phi_{1}\left[\begin{array}{c}
z, q \\
z q
\end{array} ; q\right]
$$


Theorem 4.7.1. When $a=1$, the Nevanlinna matrix is given by

$$
\begin{gathered}
A^{(1)}(z)=-[q]_{\infty} \sum_{n=1}^{\infty}\left((1+z) h_{n}(1+z)+2 q[1+z]_{n} g_{n}(q)\right) \frac{q^{n}}{[q]_{n}^{2}}-a_{q}[q]_{\infty} C^{(1)}(z), \\
B^{(1)}(z)=\frac{1}{[q]_{\infty}}(1+z) h_{\infty}(1+z)-\xi_{0} D^{(1)}(z), \\
C^{(1)}(z)={ }_{2} \phi_{1}\left[\begin{array}{c}
1+z, 0 \\
q
\end{array} ; q\right], \quad D^{(1)}(z)=-\frac{[1+z]_{\infty}}{[q]_{\infty}^{2}}
\end{gathered}
$$

where

$$
\begin{gathered}
a_{q}=\sum_{n=0}^{\infty} \frac{q^{n}}{[q]_{\infty}\left(1-q^{n}\right)}=\frac{q}{(1-q)^{2}}{ }_{3} \phi_{2}\left[\begin{array}{c}
q, q, 0 \\
q^{2}, q^{2}
\end{array} ; q\right] \\
\xi_{0}=[q]_{\infty}\left(a_{q}+2 q g_{\infty}(q)-1\right) .
\end{gathered}
$$

For $t=-1 / \xi_{0}$, the corresponding $\mathrm{N}$-extremal measure $\nu_{t}^{(1)}$ is concentrated in the zeros of $(1+z) h_{\infty}(1+z)$, which are $x_{-1}=-1$ and $\left(x_{n}-1\right)_{n \geq 0}$, where $x_{n}$ is the unique zero in ] $q^{-n}, q^{-n-1}$ [ of the function $g_{\infty}$. It does not seem possible to find $x_{n}$ explicitly.

In analogy with Proposition 4.6.1, we get:

Proposition 4.7.2. For $a=1$, we have the following one-parameter family of analytic densities for the corresponding moment problem

$$
\nu(x ; \rho)=\frac{[q]_{\infty}^{3}}{\pi} \frac{\rho}{\left((1+x) h_{\infty}(1+x)\right)^{2}+\rho^{2}[1+x]_{\infty}^{2}}, \quad 0<\rho<\infty .
$$

4.8. The determinate case. The parameter range in the determinate case consists of two disjoint regions

(1) $a \leq q$,

(2) $1 / q \leq a$,

and we shall find the orthogonality measure $\nu^{(a)}$ in the two cases.

Let us first consider the case $a \leq q$. The generating functions (4.10), (4.11) have a simple pole at $w=1 / q$, and this is the singularity closest to zero; the second closest is $1 / q^{2}$ which is a simple pole if $a<q$ and a double pole if $a=q$. By considering the principal part of the generating functions, we find as in Section 4.3

$$
\begin{gathered}
F_{n}(z)=\frac{q^{n}}{[q]_{\infty}} \frac{[1+z]_{\infty}}{[a]_{\infty}}+O\left(n q^{2 n}\right) \\
\frac{1}{\mu_{1}} \tilde{F}_{n-1}(z)=\frac{q^{n}}{1-a}{ }_{2} \phi_{1}\left[\begin{array}{cc}
1+z, 0 \\
a q
\end{array}\right]+O\left(n q^{2 n}\right),
\end{gathered}
$$

where $O\left(n q^{2 n}\right)$ can be replaced by $O\left(q^{2 n}\right)$ if $a<q$. By Markov's Theorem (cf. [31], [3]), we know that

$$
\int \frac{d \nu^{(a)}(u)}{z-u}=\lim _{n \rightarrow \infty} \frac{Q_{n}(z)}{P_{n}(z)}=-\lim _{n \rightarrow \infty} \frac{1}{\mu_{1}} \frac{\tilde{F}_{n-1}(z)}{F_{n}(z)} \text { for } z \in \mathbb{C} \backslash \mathbb{R},
$$


and by (4.34) we find

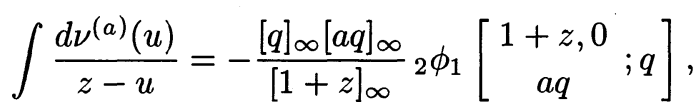

which is precisely the same expression as (4.26) with $\varphi=0$, but with $a, q$ in another parameter range. The computation of $\nu^{(a)}$ is therefore exactly as the computation of $\nu_{0}^{(a)}$ in Section 4.5 and leads to the same formula.

In case (2), a similar computation leads to the desired measure, but we can also use the invariance properties (4.7) of $F_{n}^{(a)}$ and $\tilde{F}_{n}^{(a)}$ under the transformation $a \mapsto 1 / a$. This gives for $1 / a \leq q, z \in \mathbb{C} \backslash \mathbb{R}$ :

$$
\begin{aligned}
\int \frac{d \nu^{(a)}(u)}{z-u} & =-\lim _{n \rightarrow \infty} \frac{1}{\mu_{1}} \frac{\tilde{F}_{n-1}^{(a)}(z)}{F_{n}^{(a)}(z)} \\
& =-\lim _{n \rightarrow \infty} \frac{1}{\mu_{1}} \frac{a^{n-1} \tilde{F}_{n-1}^{(1 / a)}((1+z) / a-1)}{a^{n} F_{n}^{(1 / a)}((1+z) / a-1)} \\
& =-\frac{1}{a} \frac{[q]_{\infty}[q / a]_{\infty}}{[(1+z) / a]_{\infty}}{ }_{2} \phi_{1}\left[\begin{array}{c}
(1+z) / a, 0 \\
q / a
\end{array} ; q\right]
\end{aligned}
$$

which is precisely the same expression as (4.26) with $\varphi=-1 / \xi(a)$, so $\nu^{(a)}$ is formally the same as $\nu_{-1 / \xi(a)}^{(a)}$, but for $a$ in another parameter range.

Summing up, we have

Theorem 4.8.1. In the determinate case the unique orthogonality measure is given by

$$
m^{(a)}=[a q]_{\infty} \sum_{n=0}^{\infty} \frac{a^{n} q^{n^{2}}}{[a q]_{n}[q]_{n}} \varepsilon_{\left(q^{-n}-1\right)} \quad \text { for } a \leq q
$$

and by

$$
\sigma^{(a)}=[q / a]_{\infty} \sum_{n=0}^{\infty} \frac{a^{-n} q^{n^{2}}}{[q / a]_{n}[q]_{n}} \varepsilon_{\left(a q^{-n}-1\right)} \quad \text { for } 1 / q \leq a .
$$

4.9. The moments. For $0<q<1$, let $G_{n}(a)=G_{n}(a ; q)$ be the polynomial in $a$ of degree $n$ determined by the recursion

$$
G_{n+1}(a)=(1+a) G_{n}(a)-a\left(1-q^{-n}\right) G_{n-1}(a), \quad n \geq 0,
$$

and the initial conditions

$$
G_{-1}=0, \quad G_{0}=1
$$

As shown in [2], we have

$$
G_{n}(a)=\sum_{k=0}^{n}\left[\begin{array}{l}
n \\
k
\end{array}\right] q^{k(k-n)} a^{k}
$$

where

$$
\left[\begin{array}{c}
n \\
k
\end{array}\right]=\frac{[q]_{n}}{[q]_{k}[q]_{n-k}}
$$


Al-Salam and Carlitz noticed in [2] that

$$
u_{n}^{*}(a)=\sum_{k=0}^{\infty} \frac{q^{-n k} q^{k^{2}} a^{k}}{[a q]_{k}[q]_{k}}, \quad n \geq 0,
$$

satisfies (4.36). In fact, $u_{n}^{*}(a)$ is well-defined for $a \neq q^{-n}, n \geq 1$, and for these $a$ we find

$$
\begin{aligned}
u_{n+1}^{*}(a)-(1 & +a) u_{n}^{*}(a)+a\left(1-q^{-n}\right) u_{n-1}^{*}(a) \\
= & \sum_{k=0}^{\infty} \frac{q^{-n k} q^{k^{2}} a^{k}}{[a q]_{k}[q]_{k}}\left\{q^{-k}\left(1-q^{k}\right)\left(1-a q^{k}\right)-a q^{-n+k}\right\}=0 .
\end{aligned}
$$

Furthermore, it can be proved by induction that

$$
\sum_{k=0}^{n} \frac{q^{k(k-1)}-(1+a) q^{k^{2}}}{[a q]_{k}[q]_{k}} a^{k}=-\frac{q^{n(n+1)} a^{n+1}}{[a q]_{n}[q]_{n}}, \quad n \geq 0,
$$

which for $n \rightarrow \infty$ yields $u_{1}^{*}(a)=(1+a) u_{0}^{*}(a)$ since the right-hand side tends to zero. It follows that

$$
u_{n}^{*}(a)=u_{0}^{*}(a) G_{n}(a), \quad n \geq 0 .
$$

From Proposition 4.5.1 and Theorem 4.8.1, we know that $u_{0}^{*}(a)=1 /[a q]_{\infty}$ when $0<a<1 / q$, thereby showing that the measure

$$
m^{(a)} * \varepsilon_{1}=[a q]_{\infty} \sum_{k=0}^{\infty} \frac{a^{k} q^{k^{2}}}{[a q]_{k}[q]_{k}} \varepsilon_{q^{-k}}, \quad 0<a<1 / q
$$

has the moment sequence $\left(G_{n}(a)\right)_{n \geq 0}$. The measure (4.40) is the translation of (1.6) by 1 .

As noticed in [2], the translation of (1.7)

$$
\sigma^{(a)} * \varepsilon_{1}=[q / a]_{\infty} \sum_{k=0}^{\infty} \frac{a^{-k} q^{k^{2}}}{[q / a]_{k}[q]_{k}} \varepsilon_{a q^{-k}}, \quad q<a
$$

also has the moment sequence $\left(G_{n}(a)\right)_{n \geq 0}$. In fact, the $n$-th moment of $\sigma^{(a)} * \varepsilon_{1}$ is

$$
\begin{aligned}
a^{n}[q / a]_{\infty} \sum_{k=0}^{\infty} \frac{q^{-n k} q^{k^{2}} a^{-k}}{[q / a]_{k}[q]_{k}} & =a^{n} \int x^{n} d\left(m^{(1 / a)} * \varepsilon_{1}\right)(x) \\
& =a^{n} G_{n}(1 / a)=G_{n}(a) .
\end{aligned}
$$

Let $s_{n}(a)=s_{n}(a ; q)$ denote the $n$-th moment corresponding to the birth and death rates (4.1) for $0<q<1,0<a$. By (4.40) and (4.41), the moments $s_{n}(a)$ and $G_{n}(a)$ are connected via the formulas

$$
G_{n}(a)=\sum_{k=0}^{n}\left(\begin{array}{l}
n \\
k
\end{array}\right) s_{k}(a), \quad s_{n}(a)=\sum_{k=0}^{n}\left(\begin{array}{l}
n \\
k
\end{array}\right)(-1)^{n-k} G_{k}(a) .
$$

Lemma 4.9.1. For $a>0$ and $0<q<1$, we have

(i) $\sqrt[n]{s_{n}(a)} \leq \sqrt[n]{G_{n}(a)} \leq \sqrt[n]{s_{n}(a)}+1$

(ii) $q^{1 / 4} \sqrt{a} q^{-n / 4} \leq \sqrt[n]{G_{n}(a)} \leq(1+a) q^{-n / 4}$. 
Proof. From Hölder's inequality we get

$$
s_{k}(a) \leq s_{n}(a)^{k / n} \text { for } 0 \leq k \leq n,
$$

so, by the first equation in (4.42), we obtain

$$
s_{n}(a) \leq G_{n}(a) \leq \sum_{k=0}^{n}\left(\begin{array}{l}
n \\
k
\end{array}\right) s_{n}(a)^{k / n} \leq\left(1+\sqrt[n]{s_{n}(a)}\right)^{n}
$$

which shows (i).

To see the first inequality in (ii), we use (4.38). For $n=2 p$, we have

$$
G_{2 p}(a) \geq\left[\begin{array}{c}
2 p \\
p
\end{array}\right] q^{-p^{2}} a^{p} \geq q^{-n^{2} / 4} a^{n / 2}
$$

and for $n=2 p+1$,

$$
\begin{aligned}
G_{2 p+1}(a) & \geq\left[\begin{array}{c}
2 p+1 \\
p
\end{array}\right] q^{-p(p+1)} a^{p}+\left[\begin{array}{c}
2 p+1 \\
p+1
\end{array}\right] q^{-p(p+1)} a^{p+1} \\
& \geq q^{\left(1-n^{2}\right) / 4} a^{n / 2} \frac{1+a}{\sqrt{a}} \geq 2 q^{1 / 4} q^{-n^{2} / 4} a^{n / 2}
\end{aligned}
$$

For the second inequality in (ii), we use

$$
q^{k(k-n)} \leq q^{-n^{2} / 4} \text { and }\left[\begin{array}{l}
n \\
k
\end{array}\right] \leq\left(\begin{array}{l}
n \\
k
\end{array}\right) \text { for } 0 \leq k \leq n,
$$

and find from (4.38)

$$
G_{n}(a) \leq q^{-n^{2} / 4} \sum_{k=0}^{n}\left(\begin{array}{l}
n \\
k
\end{array}\right) a^{k}=(1+a)^{n} q^{-n^{2} / 4} .
$$

Corollary 4.9.2. The Carleman series

$$
\sum_{n=0}^{\infty} \frac{1}{\sqrt[2 n]{s_{n}(a)}}<\infty
$$

for all $a>0$ and $0<q<1$.

Remark 4.9.3. The above Corollary shows that Carleman's criterion is too rough to distinguish between determinacy and indeterminacy in the parameter domain $\Omega$.

The Darboux method can be used to obtain precise information about the asymptotic behaviour of $G_{n}(a)$ because of the following generating function for $G_{n}^{2}(a)$

$$
\sum_{n=0}^{\infty}(-1)^{n} q^{n(n+1) / 2} G_{n}^{2}(a) \frac{w^{n}}{[q]_{n}}=\frac{[q w]_{\infty}[a q w]_{\infty}^{2}\left[a^{2} q w\right]_{\infty}}{\left[q a^{2} w^{2}\right]_{\infty}}
$$

This formula is a special case of (1.10) in [2]. The function $\varphi(w)$ on the right-hand side is meromorphic, and the singularities with smallest absolute value are $w= \pm(a \sqrt{q})^{-1}$ with

$$
\operatorname{Res}\left(\varphi, \pm \frac{1}{a \sqrt{q}}\right)=\frac{[ \pm \sqrt{q} / a]_{\infty}[ \pm \sqrt{q}]_{\infty}^{2}[ \pm a \sqrt{q}]_{\infty}}{ \pm 2 a \sqrt{q}[q]_{\infty}} .
$$

The point $w=-(a \sqrt{q})^{-1}$ is a simple pole, but if $a=q^{n+1 / 2}$ for some $n \in \mathbb{Z}$, then $w=(a \sqrt{q})^{-1}$ is a removable singularity. 
By the Darboux method, we get

$$
G_{n}(a)=\frac{a^{n / 2} q^{-n^{2} / 4}}{\sqrt{\left[q^{n+1}\right]_{\infty}}} \rho_{n}\left(1+O\left(q^{n / 2}\right)\right),
$$

where $\rho_{n}>0$ is given by

$$
2 \rho_{n}^{2}=\left[-\frac{\sqrt{q}}{a}\right]_{\infty}[-\sqrt{q}]_{\infty}^{2}[-a \sqrt{q}]_{\infty}+(-1)^{n}\left[\frac{\sqrt{q}}{a}\right]_{\infty}[\sqrt{q}]_{\infty}^{2}[a \sqrt{q}]_{\infty}
$$

\section{References}

1. N. I. Akhiezer, The Classical Moment Problem, Oliver and Boyd, Edinburgh, 1965.

2. W. A. Al-Salam and L. Carlitz, Some orthogonal q-polynomials, Math. Nachr. 30 (1965), 47-61.

3. C. Berg, Markov's Theorem revisited, J. Approx. Theory, to appear.

4. C. Berg and J. P. R. Christensen, Density questions in the classical theory of moments, Ann. Inst. Fourier (3) 31 (1981), 99-114.

5. C. Berg and H. L. Pedersen, On the order and type of the entire functions associated with an indeterminate Hamburger moment problem, Ark. Mat., to appear.

6. C. Berg and M. Thill, A density index for the Stieltjes moment problem, IMACS Annals on Computing and Applied Math. 9 (1991), 185-188; Orthogonal Polynomials and Their Applications, (eds. Claude Brezinski, Laura Gori and André Ronveaux), Proceedings from the Third International Symposium on Orthogonal Polynomials, Erice 1990.

7. C. Berg and G. Valent, Nevanlinna extremal measures for some orthogonal polynomials related to birth and death processes, J. Comput. Appl. Math., Proceedings of the Evian Conference, October 1992, to appear.

8. H. Buchwalter and G. Cassier, Mesures canoniques dans le problème classique des moments, Ann. Inst. Fourier (2) 34 (1984), 45-52.

9. __ La paramétrisation de Nevanlinna dans le problème des moments de Hamburger, Exposition. Math. 2 (1984), 155-178.

10. T. S. Chihara, An Introduction to Orthogonal Polynomials, Gordon and Breach, New York, 1978.

11. __ Indeterminate symmetric moment problems, J. Math. Anal. Appl. 85 (1982), 331-346.

12. T. S. Chihara and M. E. H. Ismail, Extremal measures for a system of orthogonal polynomials, J. Constructive Appr. 9 (1993), 111-119.

13. A. Erdélyi et al, Higher Transcendental Functions, 3, McGraw Hill, New York, 1953.

14. G. Gasper and M. Rahman, Basic Hypergeometric Series, Cambridge University Press, Cambridge, 1990.

15. I. S. Gradshteyn and I. M. Ryzhik, Table of Integrals, Series, and Products, Academic Press, Orlando, 1980.

16. M. E. H. Ismail, A queueing model and a set of orthogonal polynomials, J. Math. Anal. Appl. 108 (1985), 575-594.

17. M. E. H. Ismail, J. Letessier, D. Masson, and G. Valent, Birth and death processes and orthogonal polynomials. In: Orthogonal Polynomials: Theory and Practice, 229-255, NATO ASI series C 294, Kluwer Academic Publishers, The Netherlands, 1990.

18. M. E. H. Ismail and D. Masson, Q-Hermite polynomials, biorthogonal rational functions and Q-beta integrals, Memoirs Amer. Math. Soc. (1994), to appear.

19. J. Letessier and G. Valent, Some exact solutions of the Kolmogorov boundary value problem, J. Approx. Theor. Appl. 4 (1988), 97-117.

20. D. Moak, The q-analogue of the Laguerre polynomials, J. Math. Anal. Appl. 81 (1981), 20-47.

21. R. Nevanlinna, Asymptotische Entwicklungen beschränkter Funktionen und das Stieltjessche Momentenproblem, Ann. Acad. Sci. Fenn. Ser. A (5) 18 (1922), 52.

22. M. Riesz, Sur le problème des moments et le théorème de Parseval correspondant, Acta Sci. Math. (Szeged) 1 (1923), 209-225.

23. H. F. Sandham, Some infinite series, Proc. Amer. Math. Soc. 5 (1954), 430-436.

24. J. Shohat and J. Tamarkin, The Problem of Moments, Amer. Math. Soc., Providence, R.I., 1943.

25. T. J. Stieltjes, Recherches sur les fractions continues, Ann. Fac. Sci. Toulouse Math. 8 (1894), $1-122 ; 9$ (1895), 5-47. 
26. M. Stone, Linear Transformations in Hilbert Space and Their Applications to Analysis, Amer. Math. Soc., New York, 1932.

27. G. Szegö, Orthogonal Polynomials, Amer. Math. Soc., Providence, R.I., 1985.

28. G. Valent, Exact solutions of a quartic birth and death process and related orthogonal polynomials, submitted.

29. - Orthogonal polynomials for a quartic birth and death process, J. Comput. Appl. Math. 49 (1993), 281-288.

30. G. Valent, Asymptotic analysis of some associated orthogonal polynomiais connected with elliptic functions, SIAM J. Math. Anal., to appear.

31. W. Van Assche, Orthogonal polynomials, associated polynomials and functions of the second kind, J. Comput. Appl. Math. 37 (1991), 237-249.

32. E. T. Whittaker and G. N. Watson, A Course of Modern Analysis, Cambridge University Press, Cambridge, 1965.

33. S. Wigert, Sur les polynômes orthogonaux et l'approximation des fonctions continues, Ark. Mat. (18) 17 (1923).

Matematisk Institut, Universitetsparken 5, DK-2100 Copenhagen O, Denmark

Laboratoire de Physique Théorique et hautes Energies, Unité associée au CNRS UA 280, Université Paris 7, 2 Place Jussieu, F-75251 Paris, France 